\begin{tabular}{|l|l|l|l|}
\hline & & $25-49$ & Hannover 2000 \\
\hline
\end{tabular}

\title{
Eine Klimakurve des Oberpleistozäns aus dem rheinischen Löss
}

\author{
WOLFGANG SCHIRMER*)
}

\author{
Es ist so gut, Ideen zu leben, \\ zu zeichnen, schreiben, sie zu weben, \\ nicht den Ideen von ungefähr und eben, \\ viel eher denen, die wie Reben \\ sich langsam windend Formen geben, \\ nach spannend erddurchgrabend Streben \\ sich leise aus dem Dunst erheben, \\ noch vaggestaltig vor uns schweben, \\ urplötzlich dann mit freudig Beben \\ aus Bruchstücken ein Ganzes geben.
}

Schirmer, W. (2000): Eine Klimakurve des Oberpleistozäns aus dem rheinischen Löss. - Eiszeitalter und Gegenwart, 50: 25-49; Hannover 2000.

Keywords: Upper Pleistocene, loess, fossil soils, climate record, ice records, deep-sea records, Niederrhein, Maas.

Kurzfassung: Eine rheinische Löss-Boden-Folge, die die letzten 130.000 Jahre umfasst, bietet an Hand von braunen und humosen Böden eine reichere Zahl von Klima-Schwankungen der letzten Kaltzeit als bisher vom terrestrischen Bereich berichtet wurde (Abb. 3, 8). Neunzehn interstadiale Böden lassen sich tendenziell, manchmal auch bis in Einzelheiten nach Alter, Intensität und Gruppierung mit den Interstadialen 1-24 (sensU DANSGAARD et al. 1993) aus grönländischen Eiskernen und mit Tiefseesedimentkernen korrelieren (Abb. 9, $10)$.

Eem und Unterwürm präsentieren sich als charakteristischer Bodenkomplex mit dreigeteilter Bodengruppe (Rocourt-Solkomplex): Ein sehr kräftiger Boden, eine Parabraunerde von Eem-Alter (Rocourt-Boden); zwei starke Böden, eine Parabraunerde (Pescher Boden), dem Brørup zugestellt, und eine Humusparabraunerde (Holzer Boden), dem Odderade zugestellt; schließlich eine Gruppe schwacher Böden, Holzer Humuszone, Titzer Boden und Titzer Humuszone, die in den Bereich der Interstadiale [=IS] 20 und 19 eingeordnet werden. Die Gruppe der schwachen Böden schließt den Rocourt-Solkomplex ab und sollte mit den IS 20 und 19 noch dem marinen Isotopenstadium (MIS) 5 zugeordnet werden. Für diese werden in Mitteleuropa Parallelen zu den Straten Dürnten, Samerberg-Dürnten,

*) Anschrift des Verfassers: Prof. Dr. W. Schirmer, Abt. Geologie der HHUniversität, Universitätsstr. 1, D-40225 Düsseldorf; schirmer@uni-duesseldorf.de
Ognon, Mittlere und Obere Mosbacher Humuszone im Rheingau, Oerel/Glinde und vielleicht Keller IV gezogen. Der Löss zwischen dem Eem-Boden und der Obergrenze der Titzer Humuszone ist der Rheingau-Löss.

Das Mittelwürm (Ahrgau-Löss) gliedert sich in vier markante Abschnitte (wm 1-4): wm 1: Mächtige LössFließserde verkörpert den ersten von zwei großen Kaltabschnitten der letzten Kaltzeit, entsprechend der Mittelwürm-Vereisung 1 (MWG 1). Inmitten liegt der braune Reisberg-Boden [? IS 18]. wm 1 entspricht dem MIS 4. wm 2: Längste Phase des Mittelwürms mit fünf braunen Interstadial-Böden (Kalkbraunerden), bestehend aus zwei Unteren Remagener Böden [IS 17-14], mit dem zweiten als stärkstem [14], und aus drei Oberen Remagener Böden [IS 12-10]. Zwischen Unteren und Oberen Remagener Böden findet sich der größste Abstand innerhalb der Remagener Bodenfolge. wm 3: Löss des Kripp-Stadials, vielleicht entsprechend der MWG 2. wm 4: Kurze Warmphase mit drei braunen Interstadial-Böden, den Sinziger Böden 1-3 [IS 8, 7, 6+5]. Die Böden nehmen an Intensität von unten nach oben ab. wm 2-4 stellt das MIS 3 dar. Diese charakteristische MittelwürmFolge bildet sich in Einzelheiten in Eis- und Tiefseesedimenten $\mathrm{ab}$.

Das Oberwürm gliedert sich in drei Abschnitte (wo 1-3): wo 1 (tieferes Hochwürm): Bevorzugt feingeschichteter Löss (Hesbaye-Löss) mit drei Erbenheimer Nassböden (E1-3). E2 [? IS 4+3]. Über dem E3-Nassboden liegt die Eltviller Tephra. Knapp darüber, bevorzugt im Niederrhein-Maas-Sambre-Raum, folgt die Eben-Diskordanz als Einebnungsfläche. Sie erzeugt eine Aufarbeitungslage älterer Lösse und Böden (KesseltLage). Im höheren Teil dieses Lösses sollte das LGM liegen. wo 2 (Höheres Hochwürm): Bevorzugt äolischer und hellster Löss mit den geringsten Verlagerungsmerkmalen (Brabant-Löss). Im Niederrhein-Maas-Ge- 
biet enthält er eine Folge von verschiedenen Nassböden und zwei terrestrischen Böden: schwach humoser Belmener Nassboden - humoser Elfgener Boden [?IS 2] - Nassboden (die drei entsprechen zusammen dem Erbenheimer Boden 4) - brauner Leonard-Boden, häufig mit Nassböden vergesellschaftet - Gustorfer Nassboden mit kräftigen Eiskeilen. wo $1+2$ vertreten als Hochglazial das MIS 2. wo 3 (Spätwürm): Auf dem obersten wo 2-Löss liegt eine basale schwache pollenanalytische Warmphase (Meiendorf), gefolgt von kurzer Abkühlung (Dryas 1), kurzer, kräftiger Warmphase (Bølling) [IS 1e], schwacher Abkühlung (Dryas 2) [IS 1d], längerer Warmphase (Allerød) [IS 1c-a] und schließlich kräftiger Abkühlung (Dryas 3). Die drei spätglazialen Warmphasen dokumentieren sich als gemeinsame Pararendzina (Mendiger Boden). Im späten Allerød ist im Mittelrheingebiet die Laacher See-Tephra verbreitet. In der Dryas 3 ist Flugsand vorherrschend.

Mit ihrer Löss-Boden-Folge zeigt die Rheinlöss-Folge die beste Korrelierung zwischen dem terrestrischen, marinen und glaziären Milieu, die bisher beobachtet wurde. Abschnittsweise zeigt die terrestrische Ausbildung eine feinere klimastratigraphische Gliederung als das marine Milieu. Die reinen Lössabschnitte in dieser Rheinlöss-Folge nehmen deutlich geringere Zeiträume ein als die bodenreichen Abschnitte. Der Gesamt-Klimahaushalt der Letzten Eiszeit stellt sich in der Rheinlöss-Folge wärmer dar als für den terrestrischen Bereich bisher angenommen.

\section{[Upper Pleistocene climate record of the Rhein loess]}

Abstract: A loess and fossil soil record of the Lower Mittelrhein and Niederrhein-Maas area covering the last 130,000 years demonstrates more variability of the terrestrial climate during the Last Glacial period than has been realized up till now (Figs. 3, 8). The succession representing the last glacial period is subdivided by 19 interstadial soils. They closely match the climate oscillations of the Greenland ice-core and the deep-sea records in age, configuration and intensity (Figs. 9, 10). The Eemian and Lower Würmian (ca. 130.000$67.000 \mathrm{a}$ BP) is represented by the Rocourt Solcomplex comprising three different soil groups: a strong luvisol, two weaker luvisols and humic soils intercalated by a faint luvisol. The strong basal luvisol (Rocourt Soil) represents the Eemian, the second luvisol (Pesch Soil) the Brørup Interstadial (IS 24-22 sensu DANSGAARD et al. 1993], the third one (Holz Soil) the Odderade Interstadial [IS 21]. The fourth (Titz Soil) sandwiched by humic soils (HH and TH) should match the region of IS 20 and 19. They form the top of the Rocourt Solcomplex of the last interglacial/early glacial period. Thus, IS 20 and 19 respectively the Titz and $\mathrm{TH}$ soils represent the top of the Early Glacial, top of marine isotope stage (MIS) 5

The Middle Würmian Ahrgau loess (ca. 67.00028.000 a BP) shows a fourfold alternation: The sequence stadial-interstadial-stadial-interstadial produces the divisions wm 1-4. wm 1: Thick solifluction loess indicates cool conditions with the short Reisberg interstadial soil [? IS 18]. $w$ m 1 represents the MIS 4 respectively the terrestrial period of the Middle Würmian Glacia- tion 1 (MWG 1). wm 2: A bundle of five brown interstadial cambisols shows a distinct configuration: two Lower Remagen Soils (R1-2) are separated from three Upper Remagen Soils (R3-5) by a short loess interval. The Remagen Soils 1-5 form the largest interstadial interval within the Middle Würmian. The second soil (R2) is the thickest and shows the highest $\mathrm{C}$ content. wm 3: A loess layer, thinner than the wm 1 loess, separates the interstadial Remagen and Sinzig Soil groups. It evidences the cooler Kripp Stadial, which may be comparable to the Middle Würmian Glaciation 2 (MWG 2). wm 4: A bundle of 3 Sinzig Soils demonstrates a short but intense interstadial group. The first interstadial soil, Sinzig 1, is the strongest one with the highest $\mathrm{C}$ content. As a whole, wm 2-4 represents the terrestric equivalent of MIS 3 and forms a conspicuous sequence likewise presented in ice and deep-see records.

The Upper Würmian (ca. 28.000-12.600 a BP) is subdivided into three units (wu 1-3): wu 1: Thick loess with tendency of fine lamination (Hesbayan Loess) is intercalated by three gleyic gelisols (Nassböden), the Erbenheim Soils (E1-3). E2 [? IS 4+3]. Above the E3 Nassboden the Eltville Tephra forms the most reliable stratigraphic layer of the Upper Würmian. The tephra is closely followed by the Eben Discordance, that widely extends over the Niederrhein-Maas-Sambre area. Its reworking activity produced a bed incorporating reworked older loesses and soils (Kesselt Layer). The upper part of the Kesselt Layer is suggested to host the LGM. wu 2: A layer up to $6 \mathrm{~m}$ thick, light coloured and preferably of eolian loess veneers the landscape (Brabantian Loess). In the Niederrhein-Maas area it encompasses several gelic gleysols (Nassböden) and two terrestric soils: Belmen Nassboden - calcaric regosol, the Elfgen Soil [?IS 2] - Nassboden (this soil trinity equals the Erbenheim Soil 4) - brown calcaric cambisol, the Leonard Soil, accompanied by Nassböden - Gustorf Nassboden with large ice wedges. wu 1-2 represents MIS 2. wu 3 (Late Würmian): On top of the wu 2 loess, the Late Glacial palynologically starts with a weak warming (Meiendorf Interstadial), followed by a short cooling (Dryas 1), a short and pointed warming (Bølling) [IS 1e], a slight cooling (Dryas 2) [IS 1d], a longer warm phase (Allerød) [IS 1c-a] and finally a strong cooling (Dryas 3). The late Allerod period hosts the Laacher See tephra. Eolian sand prevails during Dryas 3 .

Thus, the Rhein loess record for the last glacial period demonstrates the best match between terrestrial and marine/ice records reported thus far. It indicates the periods of loess deposition to cover less time than the soilforming periods. From this evidence it is argued that the climate of the last glacial stage on land was warmer than has been assumed hitherto.

\section{Vorgeschichte}

Für das Oberpleistozän Mitteleuropas wurden zahlreiche Klimakurven vorgestellt, eine ältere Gruppe zum Beispiel von Büdel (1953: 258), WOLDSTEDT (1954: 43, 1956: 82, 1958a: 151, 1958b: 244, 1960: 156, 1962: 121), MÜLLER-BECK (1959: 156), Andersen et al. (1960: 40) und Fink (1960: 


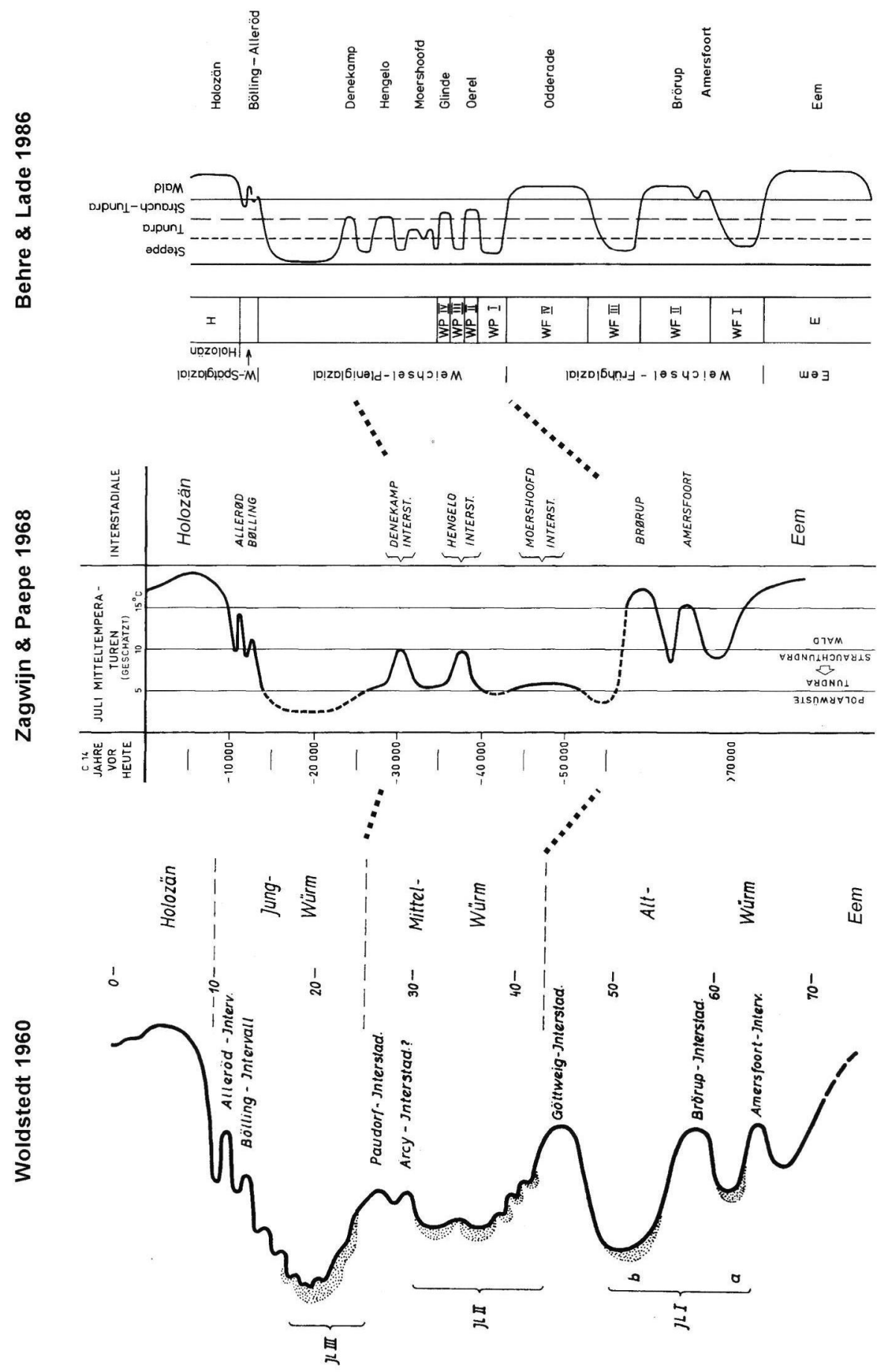

Abb. 1: Klimakurven des Oberpleistozäns von Woldstedt (1960: 156), ZAGWIJN \& Paepe (1968: 135) und Behre \& LADE (1986: 33)

Fig. 1: Upper Pleistocene climate curves of Woldstedt (1960: 156), ZaGwijn \& PAepe (1968: 135) and Behre \& Lade (1986: 33) 
263), eine jüngere Gruppe von van der Hammen et al. (1967: 92), ZaGwijn \& PAEPE (1968: 135), STAALDuinen et al. (1979: 43), VANDENBerghe (1985: 36) und Behre \& Lade (1986: 33). Drei von ihnen sind in Abb. 1 dargestellt. Die älteren sind in Abb. 1 durch die Kurve von WOLDSTEDT 1960 vertreten. Sie beinhalten zum Teil Bodenbildungen, die sich als ältere Interglazial-Böden erwiesen haben, wie die "Göttweiger Verlehmung” (BAYER 1927: 352; vgl. Fink 1964: 230), deren Warmgipfel in dieser Form im Würm gar nicht existiert. Oder es handelt sich um aufgegebene Bodennamen, wie die „Paudorfer Verlehmungszone" (Götzinger 1935: 130), die sich an ihrem locus typicus ebenfalls als Interglazial-Boden erwies (FINK 1969: 19), andernorts aber als Name für ein tatsächlich vorhandenes Interstadial im Würm verwendet wurde, nämlich eines aus der Gruppe der Mittelwürm-Interstadiale. Fink (1969: 14) empfahl an seiner Stelle die Bezeichnung Stillfried B und setzt diesen Boden dem floristisch definierten Denekamp-Interstadial auf Grund vergleichbarer stratigraphischer Position und 14C-Alter gleich (FI^K 1979: 95). Im Spätglazial dieser älteren Kurven stellen Bølling und Allerød deutliche Erwärmungsphasen dar.

Die Gruppe der jüngeren Klimakurven, in Abb. 1 durch diejenigen von ZAGWIJN \& PAEPE und Behre \& LADE vertreten, beruhen vorwiegend auf der Kenntnis paläobotanisch belegter Warmphasen im letzten Glazial.

Die gemeinsame Betrachtung der Kurven zeigt das Eem in allen Kurven einphasig. Im Unterwü rm („Altwürm”, „Frühwürm”) finden sich in der Gliederung von WoldstedT zwei Interstadiale: damals Amersfoort und Brørup genannt, später als Brørup und Odderade bezeichnet (vgl. Menke 1976: $54 \mathrm{f}$.), so auch in der Gliederung von Behre \& Lade. Durch die Subkommission für Europäische Quartärstratigraphie (CHALINE \& JERz 1984: 186) wurde das Ende des Unterwürms mit dem Ende des zweiten Würm-Interstadials bzw. mit dem Oberende der Humuszonen definiert. Das Mittelwürm enthält in der Gliederung von WoldostedT zwei, in der von Zagwijn \& PAEPE drei Interstadiale: Moershoofd, Hengelo und Denekamp. Ihnen haben BeHRE \& LADE zwei weitere vorangesetzt: Oerel und Glinde. Der Hochw ü r m-Teil des Oberwürms bleibt ungegliedert. $\mathrm{Er}$ ist die Zeit des letzten großen Eisvorstoßes der Letzten Eiszeit. Die Gliederungen zeigen erst im Spätwürm wieder die Wärmeschwankungen, die schon die WoldsteDTsche Kurve aufführt: Bølling und Allerød.
Vergleicht man diese Kurven mit fossilen Bodenfolgen im Löss des Rheingebiets und der Mittelgebirge, so finden sie sich in der Bodengliederung nach Schönhals, Rohdenburg \& Semmel (1964) annähernd wieder: Allerdings stehen dort für die beiden Wärmeschwankungen im Unterwürm drei Humuszonen zur Verfügung. Ihre Zuordnung zu den zwei Wärmeschwankungen blieb ungeklärt (Semmel 1998a). Im Mittelwürm treten passend zur Kurve von ZaGWIJN \& PAEPE - drei Böden auf: der Gräselberger Boden, der Böckinger Boden (Bibus 1989: 8) und der Lohner Boden, welch letzteren FINK (1979: 95) mit dem Denekamp-Interstadial vergleicht.

\section{Lössprofile des Niederrhein-Maas-Gebietes}

Eigene Untersuchungen der letzten 20 Jahre im Rheinland (Abb. 2) erbrachten eine detailliertere Löss-Boden-Folge des Oberpleistozäns (Abb. 3)¹. An der Basis des Oberpleistozäns liegen drei kräftige Bt-Horizonte von Parabraunerden (RocourtBoden, Pescher und Holzer Boden 2 ), der obere gefolgt von einer Humuszone, die aus zwei entkalkten Pararendzinen mit zwischenliegender Verbraunung besteht (Holzer Humuszone, Titzer Boden, Titzer Humuszone). Das Mittelwürm beherbergt neun braune Interstadialböden: Reisberg-Boden, Remagener Böden 1-5 und Sinziger Böden 1-3. Im Oberwürm liegen noch vor dem Spätwürm ein humoser (Elfgener Boden) und ein brauner Interstadialboden (Leonard-Boden). Im Spätwürm des Rheinlands sind erstmals drei Wärmeschwankungen von U. SCHIRMER (1995) nachgewiesen, die seit Bock et al. (1985) bereits in Norddeutschland bekannt sind: Meiendorf, Bølling und Allerød (vgl. U. Schirmer 1999). Bodentypologisch sind sie als ein gemeinsamer Boden, eine Pararendzina (Mendiger Boden) (W. SCHIRMER 1995a), entwickelt3.

\section{Lithologische Lösseinheiten}

Ich habe die Lössablagerungen des NiederrheinMaas-Gebietes als lithologische Körper gemäß dem Vorgehen der belgischen Lössforscher ${ }^{4}$ mit Namen der alten fränkischen Gaue belegt (W. Schirmer 1999a) (s. Abb. 2b). - dies, um die zahlreichen Lösseinheiten lithostratigraphisch ansprechen zu können, was bisher nicht möglich war. Überdies ist die chronostratigraphische Zuordnung der präwürmzeitlichen Lösse teilweise unsicher; auch Ausschnitte des Würmlösses lassen sich in stärker von Diskordanzen geprägten Profilen oft nur den größeren würmzeitlichen Lössabschnitten zuordnen. Im übrigen ist es nicht sinn- 

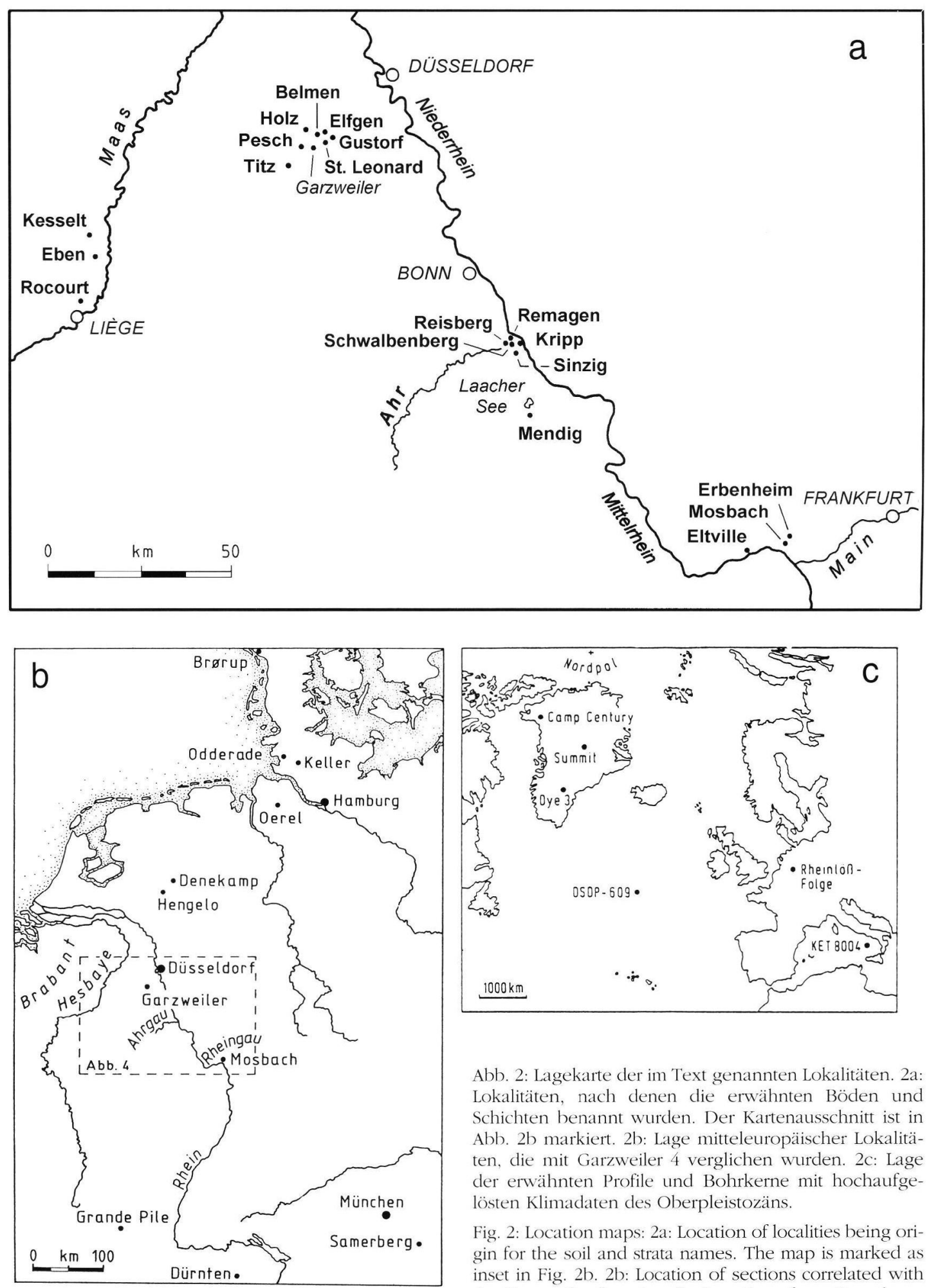

Abb. 2: Lagekarte der im Text genannten Lokalitäten. 2a: Lokalitäten, nach denen die erwähnten Böden und Schichten benannt wurden. Der Kartenausschnitt ist in Abb. 2b markiert. 2b: Lage mitteleuropäischer Lokalitäten, die mit Garzweiler 4 verglichen wurden. 2c: Lage der erwähnten Profile und Bohrkerne mit hochaufgelösten Klimadaten des Oberpleistozäns.

Fig. 2: Location maps: 2a: Location of localities being origin for the soil and strata names. The map is marked as inset in Fig. 2b. 2b: Location of sections correlated with the Garzweiler 4 section. 2c: Location of mentioned sections and cores with high-resolution climate records of the Upper Pleistocene. 
voll, die terrestrischen Ablagerungen mit Bezeichnungen der marinen oder glaziären EiskernStratigraphie zu belegen („etwa MIS 6-Löss"). Außerdem erweist sich die terrestrische Löss-Stratigraphie, wie im Folgenden gezeigt wird, in einigen Bereichen feingliedriger als die marine oder glaziäre.

Der letztglaziale Löss von der Obergrenze des Rocourt-Bodens (Abb. 3) bis zur Obergrenze der obersten Humuszone ist als Rheingau-Löss benannt. Der Löss von der Obergrenze der obersten Humuszone bis zur Obergrenze des Sinziger Bodens 3 ist der Ahrgau-Löss. Der Löss von dort bis zur Untergrenze des Belmener Bodens, also bis vor den neuen kalkreichen Lösseinsatz in der Eben-Zone, ist der Hesbaye-Löss. Der Löss von der Basis des Belmener Bodens bis zur heutigen Oberfläche ist der Brabant-Löss5

\section{Nassboden-Typen}

Die Lösseinheiten des Niederrheins sind in stärkerem Maße durch Nassböden gekennzeichnet, als das von anderen Lössgebieten Mitteleuropas berichtet wurde. Profilmorphologisch lassen sich dabei zwei Grundtypen ausscheiden: ein Gefleckter Nassboden (Ng), der graue, rostgeränderte Flecken zeigt, und ein Grauer Nassboden, der homogene Graufärbung (Nr) zeigt, dazu bevorzugt randliche Rostbänder oder auch Rostfleckung (No). Der Gefleckte Nassboden repräsentiert einen Haftnässepseudogley-Typ, der Graue Nassboden einen Gley-Typ. Beide Typen können auch in Übergangsformen (z. B. Nrg) auftreten. Da es sich bei den Nassböden, im Gegensatz zu den echten Gleyen und Pseudogleyen, um Hydromorphie auf stauendem Permafrost handelt, hat sich für die Nassböden-Typen die gemeinsame Verwendung des Symbols N (von Nassboden) als sehr hilfreich erwiesen (W. SCHIRMER 1990, 1991: 73, 1992, 1995b: 541, 1999a, b, FELDMANN \& Scinrmer 1992, Feldmann 1996: 189, Henze 1998). Die Zusatzsymbole zum Hauptsymbol N, nämlich $\mathrm{Nr}$, No und Ng, stehen dann in Analogie zu Go, Gr und $\mathrm{Sg}^{6}$.

Profiltexturell, also von der Anordnung der Böden im Profil her, gibt es wiederum zwei Nassboden-Typen: Synchrone und diachrone Nassböden.

Die synchronen kennzeichnen - wie die meisten fossilen Böden - eine gewisse Ruhephase innerhalb des Sedimentaufwuchses. Das Sediment unter dem Boden ist also älter als der Boden, dasjenige darüber jünger. Synchrone Nassböden kön- nen durch beide morphologischen Bodentypen, Gefleckte wie Graue Nassböden, vertreten werden.

Die diachronen Nassböden dagegen können wesentlich jünger als der angrenzende liegende und hangende Schichtverband sein, auch jünger als mächtige überlagernde Schichten. Ihre bisher beobachteten Kennzeichen sind folgende': Sie sind stets als Graue Nassböden ausgebildet. Sie treten gerne in Scharen zu schmalen cm- bis dm-starken Bändern auf. Sie schneiden den Schichtverband unter flachen Winkeln; das zu erkennen braucht allerdings manchmal viele Meter bis Dekameter horizontaler Verfolgung. Sie werden nur oben von schmalem Rostband begrenzt. Ihr Grau ist oben am stärksten und verklingt sanft nach unten.

Es scheint, als ob innerhalb einer Schar diachroner Böden der oberste der älteste und der unterste der jüngste ist. So könnten sie Stillstandsphasen niederschmelzenden Permafrostes im Boden markieren. Diachrone Nassböden sind in den Abbildungen dieser Arbeit nicht mit dargestellt, da sie nicht Bestandteil klimastratigraphischer Sediment-Boden-Sequenzen sind, die hier erfragt werden.

\section{Detailgliederung des Oberpleistozäns und Vergleich mit Klimakurven des Eises und der Tiefsee}

Da hier für die Detailprofile, die den Untersuchungen zugrunde liegen, nicht Platz ist, werden stellvertretend die Böden des Eems und Unterwürms (Rocourt-Solkomplex) durch das Profil Garzweiler 4 (Abb. 5 und 6) und die Böden des Mittelwürms durch das Profil des Schwalbenbergs (Abb. 7) vertreten. Ansonsten werden die Einzelglieder des Oberpleistozäns chronologisch besprochen. In Abb. 8 sind sie zu einer Klimakurve umgeformt, die sich auf lithologische Klimaindikatoren, wie z. B. Löss, auf Bodentypen und Floren- und Faunenangaben aus Straten und Böden stützt.

\subsection{Eem und Unterwürm - der gespreizte Rocourt-Solkomplex}

Im niederrheinischen Löss ist an der Basis des letztglazialen Würmlösses normalerweise der BtHorizont einer einzigen Parabraunerde mit hangendem Bleichhorizont und darüberliegender schwach gedoppelter Humuszone erhalten. Die solchermaßen gegliederte Bodenfolge wurde am Niederrhein erstmals von SCHIRMER \& STREIT (1967: 


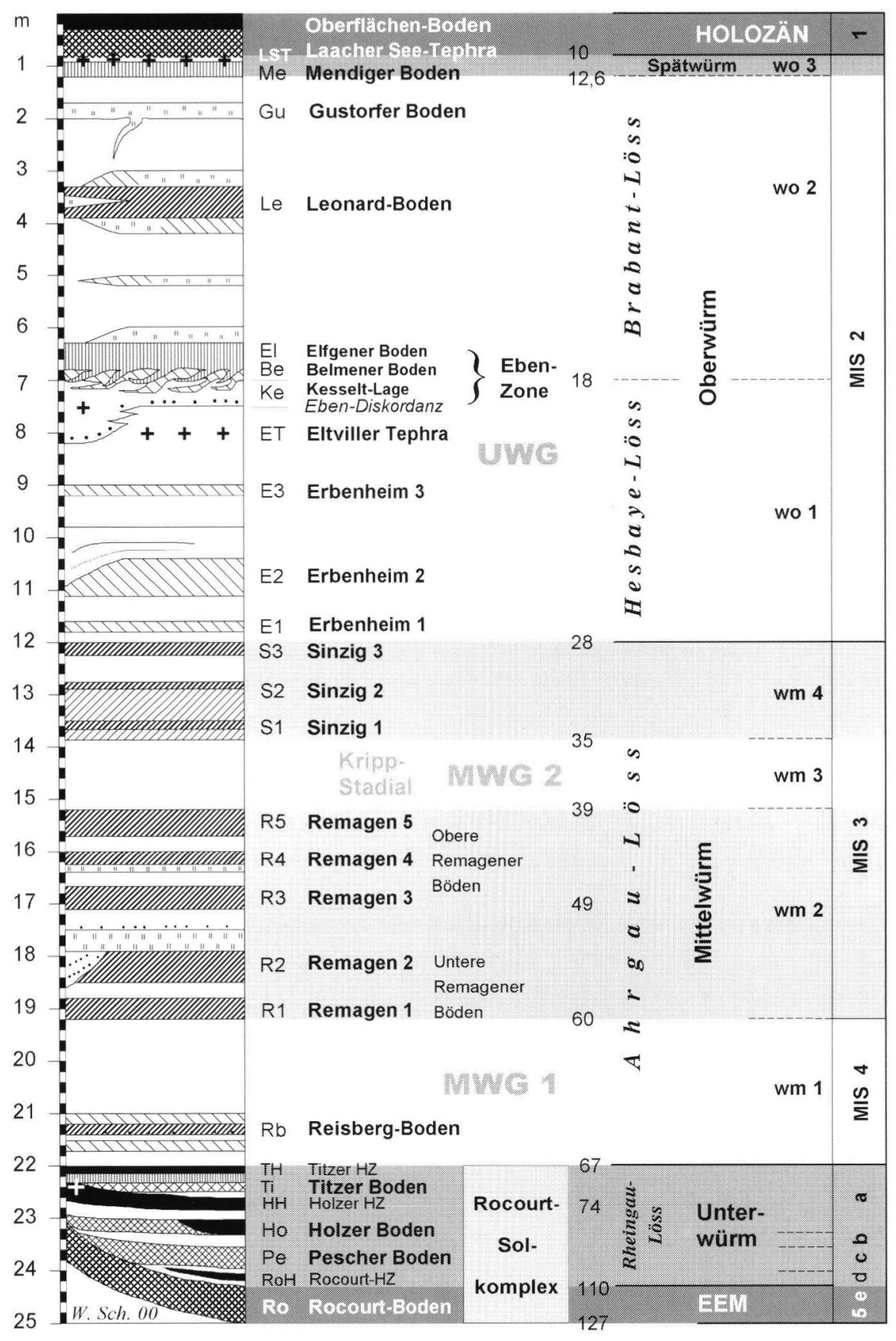

Abb. 3: Löss-Boden-Folge des Oberpleistozäns im Niederrhein-Maas-Gebiet. Von links nach rechts: Die Metrierung stellt eine mittlere vorgefundene Mächtigkeit dar; Profil-Signaturen siehe Abb. 4; leitende Böden und Sedimentlagen und deren Kurzbezeichnungen; wichtige Altersdaten in ka BP, für das Unter- und Mittelwürm nach DANSGAARD et al. (1993) und BOND et al. (1993); kursiv: lithologische Lösseinheiten (nach W. Schirmer 1999a); Gliederungseinheiten des Oberpleistozäns; marine Isotopen-Stadien (MIS) = Sauerstoff-Isotopen-Stadien (OIS). HZ = Humuszone.

Fig. 3: The Upper Pleistocene Rhein-Maas loess-soil sequence. From left to right hand: The meter scale represents an average thickness as far exhibited; for symbols see Fig. 4; characteristic soils and deposits and their abbreviations; some important ages in ka BP; italics: lithological loess units (after W. Schirmer 1999a); subunits of the Upper Pleistocene; marine isotopic stages (MIS) = oxygen isotope stages (OIS). HZ = humus zone. 
Böden der Grenze i/j) beschrieben. Gullentops (1954) hatte den Boden genau derselben Ausbildung im belgischen Maas-Gebiet bereits als Sol de Rocourt bezeichnet. Dieser Name wird, da es sich bis ins Detail um dieselbe Bodenfolge handelt, übernommen ${ }^{8}$.

In morphologischen Hohlformen kann dieser Bodenkomplex stärker auseinandergezogen sein, und zwar in vier Parabraunerden, die von unten nach oben an Intensität abnehmen und zum Teil nachfolgende Humuszonen tragen. Solche Situationen stammen vom Tagebau Garzweiler. In einem $220 \mathrm{~m}$ langen Wandanschnitt des Aufschlusses Tagebau Garzweiler 4 (Abb. 5) durchzieht im Bereich der Hauptterrassen-Hochfläche ein derart gespreizter Bodenkomplex zwei Dellentälchen, die durch einen Plateausporn getrennt sind. Der unterste der vier Bt-Horizonte, der Rocourt-Boden (Ro)9, zieht als weitaus kräftigster und rötester über Plateauposition wie Dellenhang und Dellenböden hinweg. Im Aufschluss Garzweiler 12 war über ihm eine Humuszone ( $\mathrm{RoH})$ erhalten. Die folgenden beiden, jeweils durch Löss getrennten Bt-Horizonte sind als Pescher Boden (Pe) und Holzer Boden (Ho) benannt (Abb. 2a und 5). Der Pescher Boden tritt nur in Dellenposition auf und ist dort deutlich durch Löss vom Rocourt-Boden abgesetzt. Der Holzer Boden ist fast immer diskordant über dem Rocourt-Boden als rötlichgraubrauner Bht-Saum vorhanden. In Hohlformen rückt er räumlich vom Rocourt- und Pescher Boden ab. Über dem Holzer Boden liegt, wiederum durch Löss getrennt, weitflächig erhalten, eine geschlossene schwarzbraune Humuszone, die im Normalfall farblich eine Zweiteilung erkennen lässt. Der untere Teil wird als Holzer Humuszone (HH) bezeichnet, da sie auf den Holzer Boden folgt. Lokal war zwischen ihr und dem oberen Teil der Humuszone im Aufschluss Garzweiler 12 noch ein schwacher Verbraunungshorizont (Btv), der Titzer Boden (Ti), abgesetzt. Über ihm folgt der obere Teil der Humuszone, Titzer Humuszone (TH) benannt ${ }^{10}$. Diese normalerweise gedoppelt erscheinende Humuszone schließt die Bodenfolge nach oben ab. Insgesamt wird der Boden-Komplex aus den vier Bt-Horizonten samt Humuszonen als Rocourt-Solkomplex bezeichnet.

Der basale Bt-Horizont, der Rocourt-Boden (Ro), zeigt die stärkste Gelb-Rotfärbung, der Pescher Boden (Pe) ist dagegen dunkler, rotbraun. Der Holzer Boden (Ho) ist graubraun, aber noch mit rötlichem Stich, und hat ein Polyeder-Gefüge mit
Ton- und Humusbelägen (Bht-Horizont), stellt also eine Humusparabraunerde dar. In tiefster Muldenposition kann er Pseudotschernosem-Charakter annehmen. Die drei Parabraunerden nehmen von unten nach oben an Mächtigkeit ab, dagegen an Kohlenstoffgehalt zu (Abb. 6). Der Pescher Boden nimmt eine Mittelstellung im Humusgehalt zwischen dem Rocourt-Boden und dem Holzer Boden ein. Der Titzer Boden bedarf noch der Analytik. Die Titzer Humuszone (TH) hat einen höheren C-Gehalt als die Holzer.

Einstufung: Da Altersdaten und Floren im Tagebau Garzweiler fehlen, erfolgt die Einstufung des Rocourt-Solkomplexes durch regionalen Vergleich ihrer typischen, eigencharakteristischen Warm-Kalt-Folge. Die Böden verkörpern Warmschwankungen sehr unterschiedlicher Wertigkeit mit dazwischenliegenden Abkühlungsphasen. Die Intensitäten der Warmschwankungen lassen folgendes Warmphasen-Schema erkennen:

$\mathrm{HH}+\mathrm{Ti}+\mathrm{TH}$ : Ein Gruppe schwach ausgebildeter Warmphasen

Pe+Ho: $\quad$ Zwei kräftigere und breiter ausgebildete Warmphasen

Ro: $\quad$ Eine stärkste und am breitesten ausgebildete Warmphase.

Es sei betont, dass die schwachen Warmphasen $\mathrm{HH}$, Ti und TH in dieser Form im ganzen RheinMaas-Gebiet mit erstaunlicher Mächtigkeitskonstanz von ca. $50 \mathrm{~cm}$ im Mittel $(30-70 \mathrm{~cm}$ im Extrem) und stets in derselben gleichbleibenden und vollständigen Horizontfolge auftreten. Sie bilden also, da der Titzer Boden in seiner Erscheinungsform hinter den Humuszonen zurücktritt, zwei dicht übereinander sitzende schmale und markante Horizonte.

Vergleicht man dieses Warmphasen-Schema mit Klimakurven des grönländischen Inlandeises und solchen von Tiefseesedimenten, so lassen sich darin erstaunliche Gemeinsamkeiten erkennen. Zum Vergleich wurden hier der Bohrkern GRIP Summit (Abb. 9, 10) und Tiefseekerne aus dem nördlichen Atlantik und der Tyrrhenis (Abb. 9; Lage in Abb. 2c) ausgewählt. Auch in diesen Kurven findet sich eine stärkste und am breitesten ausgebildete Warmphase als Eem, entsprechend dem marinen Isotopen-Stadium (MIS) 5e. Es folgen zwei noch kräftig und breit ausgebildete, aber wenig schwächere Warmphasen als MIS $5 c$ und a (Brørup und Odderade), entsprechend den Interstadialen (IS) 24-22 und 21 im Sinne von DansGAARD et al. (1993: 218). Den Abschluss bilden zwei schwache und schmal ausgebildete Warm- 
Ah-Horizont

oder:

Ah-Horizont, stark/mittel/schwach

x-Horizont: bioturbat durchwühlt

S(e)w-Horizont

Sd-Horizont

Bt- und Bht-Horizont

Bbv-Horizont

Bv-Horizont, auch karbonatisch (Bcv) kräftiger/schwächer

Nassböden (N-Horizont):

Ng-Horizont: haftnässefleckig

No-Horizont: rostfleckig

No-Horizont: rostgebändert

Nr-Horizont: flächenhaft grau

M-Horizont: Bodensediment

Sand

$++\quad$ Tephra

\section{$+$}

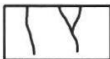

humusgefültte

Trockenrisse

Abb. 4: Legende zu den Abb. 3 und 6

Fig. 4: Legend to Figs. 3 and 6

phasen, die sich aber scharf und markant abbilden, die Interstadiale (IS) 20 und 19.

In diesem Vergleich entspricht also der RocourtBoden dem ungeteilten Interglazial $5 \mathrm{e}$. Der Pescher Boden repräsentiert $5 c$, der Holzer Boden 5a. Die schwachen, aber scharf gezogenen Böden über dem Holzer Boden sollten dann der Gruppe der Interstadiale 20 und 19 zugeordnet werden, die unmittelbar über $5 \mathrm{a}$ in zahlreichen Eiskernund Tiefseekurven auftreten. Diese beiden Interstadiale liegen an der Grenze der MIS 4 und 5 und werden von verschiedenen Autoren der einen oder anderen Stufe zugestellt. DansGaARD et al.
(1993: 219) stellen sie ins MIS 4, PISIAS et al. (1984: 124), McManus et al. (1994) stellen sie in den Übergang MIS 4/5, die meisten Autoren allerdings stellen sie in das MIS 5, wie zum Beispiel DansGAARD et al. (1971), WollLARD (1979) und PATERNE et al. (1986).

Im terrestrischen Bereich wird man in jedem Falle mit der Humuszone, die das Oberende der Bodenfolge des Rocourt-Solkomplexes markiert, das Unterwürm nach oben enden lassen und sie damit in das MIS 5 stellen. Allerdings fallen dann drei Interstadiale mit Verbraunung über dem Eem ins Unterwürm; und wenn man den Verbraunungshorizonten noch jeweils ihre Humuszonen hinzusetzt, sind es mit der Rocourt-Humuszone (RoH) sieben Interstadiale im Unterwürm. So müsste die Definition des Unterwürms der Subkommission für Europäische Quartärstratigraphie (Chaline \& Jerz 1984: 186), die nur zwei Interstadiale, aber auch die Humuszonen darin vorsieht, dann um etliche Bodeninterstadiale nach oben erweitert werden. Ansonsten fielen die höheren dieser Interstadiale dem tiefsten Mittelwürm vor der großen wm1-Kaltphase zu. Nach dem Bild jedoch, das die Natur in den Humuszonen liefert, und nach den dazugehörigen Klimaindikatoren sollten die nach jedem Interglazial auftretenden Humuszonen dem ausklingenden interglazialen Bodenkomplex zugeschlagen werden, der natürlicherweise auf die neue Glazialperiode hinweist. Die Humuszonen sollten also im Falle des letzten Interglazial/Glazial-Wechsels zum Unterwürm gezogen werden. So war auch das Unterwürm bisher verstanden worden ${ }^{11}$.

Werden die schwächeren ausklingenden Interstadiale $\mathrm{HH}$, Ti und $\mathrm{TH}$ noch in das Unterwürm gestellt, dann beginnt das Mittelwürm (wm 1) auch mit der bekannten kräftigen Kaltphase, die sich im Ozean als MIS 4 präsentiert, im Rheinland als mächtiger Löss verbreitet ist und mancherorts auch durch Hinweise auf einen offenbar bedeutenden Gletschervorstoß bekannt wurde, so Z. B. durch MaKowska (1975) in Polen, LundQvisT (1974) in Schweden, Petersen (1984) in Dänemark, MARKs et al. (1995) in Schleswig-Holstein, Krayss (1988), SCHLÜCIITER (1991) und Keller \& KRAYSS (1998) im nördlichen Alpenvorland und DE Beaulieu et al. (1991) im westlichen Alpenvorland. Ich bezeichne diese Kaltphase als MWG 1 (Mittel-Würm-Vergletscherung 1/Middle Würmian Glaciation 1) im Gegensatz zur schwächeren MWG 2 und zur Oberwürm-Vergletscherung (Upper Würmian Glaciation/UWG); für W kann auch Weichsel oder Wisconsin gesetzt werden. 
Vergleicht man dieses Warmphasen-Schema, das sich im Niederrhein-Maas-Gebiet so ausgeprägt wie in den Eis- und Tiefseekurven erkennen lässt, mit vollständigeren Ablagerungen des frühen Oberpleistozäns verschiedener mitteleuropäischer Lokalitäten (Lage in Abb. 2b), so findet man auch dort überraschende Übereinstimmungen.

Die Lokalität Oerel (Behre \& Lade 1986, Behre \& VAN DER PLICHT 1992) gilt bisher als die vollständigste bezüglich der Ausbildung des basalen Oberpleistozäns (GRÜGER 1989: 69). Auch hier sind drei Gruppen von Wärmegipfeln abnehmender Intensität durch Kaltphasen getrennt entwickelt. Sie werden dem Eem, dem Brørup und Odderade, und dem Oerel und Glinde zugewiesen. Auch hier stellen Oerel und Glinde deutlich schwächere, aber markante Interstadiale dar, die sich über zwei kräftigeren Interstadialen (Brørup und Odderade) und der basalen, mächtigsten Warmphase (Eem) entwickelt haben. Nach Profilentwicklung und Radiokohlenstoffdaten spräche nichts dagegen, Oerel und Glinde mit dem höheren Rocourt-Solkomplex zu korrelieren ${ }^{12}$.

In der Grande Pile (WoILlard 1979) sind ebenfalls drei Gruppen abnehmender Intensität der Wärmegipfel entwickelt (Abb. 7): mächtiges, breites Eem, kräftiges St. Germain I und St. Germain II, und schwaches Ognon I und Ognon II'13.

WeLten (1981, 1982) beschreibt aus Pollenprofilen des Schweizer Mittellandes über einem breiten Eem vier Frühwürm-Interstadiale (FWI 1-4). Die tieferen beiden, Amersfoort (FWI 1a)/Brørup (FWI 1b) als erstes und Odderade (FWI 2) als zweites, sind sehr kräftig, die höheren beiden, Dürnten (FWI 3) und ein unbenanntes (FWI 4), sind sehr schwach und eng beieinander liegend; dabei ist FWI 3 noch kräftiger als FWI 414.

Am Samerberg (GRÜGER 1989) sind vier Warmphasen gegenwärtig, ein breites Eem, kräftiges Brørup und Odderade und knapp darüber ein schwaches Dürnten.

Im Rheingau (Semmel 1998b) sind drei Verbraunungshorizonte im Löss vorhanden, dazu kräftige Humuszonen: Über dem Erbacher Boden als reifstem Boden (Parabraunerde) liegen zwei kräftige Böden, nämlich Verbraunungshorizonte unter je einer Mosbacher Humuszone (Untere und Mittlere Mosbacher Humuszonen). Darüber folgt eine klimatisch schwächere Obere Mosbacher Humuszone ohne basale Verbraunung ${ }^{15}$.

Von der Lokalität Keller in West-Schleswig-Holstein beschreibt Menke $(1976,1980)$ vier Boden- glieder in Sand und Sandlöss übereinander. Über einer Podsolparabraunerde (Keller I/Eem-Boden) folgen zwei Podsole (Keller II/Brørup und Keller III/Odderade), dann - allerdings hier durch eine Erosionsdiskordanz getrennt - ein Nanopodsol (Keller IV/Keller-Interstadial). Die Serie wird von Oberwürm-Ablagerungen abgedeckt. Ob also der Nanopodsol wirklich noch an die liegenden Böden anschließt oder, durch die Erosionsdiskordanz vom Unterwürm getrennt, in die große Mittelwürmlücke darüber fällt, lässt sich hier nicht sagen.

Vergleicht man diese Abfolgen miteinander, so ergeben sich folgende Gemeinsamkeiten: 1. Warmphase: Das Eem ist in allen Profilen der kräftigste basale Abschnitt mit den wärmsten Klimaansprüchen, ein unbestrittenes Interglazial. 2. und 3. Warmphase: Sie sind noch kräftig, aber schwächer als die erste. Die zweite Warmphase ist in Eiskernen und manchen Tiefseesedimenten schwach unterteilt; in den Pollenprofilen wird das durch Amersfoort und Brørup sichtbar. Sie ist die entschieden längere Phase von den beiden kräftigen Unterwürm-Warmphasen. Der Sedimentationsabstand zwischen Brørup und Odderade ist in sämtlichen oben genannten terrestrischen wie auch in den marinen und glaziären Profilen der größte innerhalb der oberen vier Glieder der Warmphasen. Der Abstand zwischen Odderade und den IS 20 und 19 ist in all diesen Profilen kleiner. Höhere Warmphasen: Sie folgen, soweit vorhanden, in allen Profilen sehr dicht und ohne eine merklichen Diskordanz auf die 3. Warmphase. Sie sind viel schwächer als die 2. und 3. Warmphase. Sie bilden eine dicht übereinander liegende Folge. In einigen Profilen ist nur eine dieser Warmphasen entwickelt, in anderen zwei oder drei. Hier kann nur am Ort entschieden werden, ob im Falle geringerer Anzahl im Hangenden ein Abtrag vorliegt oder die eng zusammenhängenden Warmphasen örtlich als gemeinsame, kondensierte erscheinen.

Sehr unwahrscheinlich erscheint eine Zuordnung der tieferen drei Bodenglieder zu einem dreigeteilten Eem (GRIP 1993), wenngleich eine derartige Zuordnung die Unterstützung durch die marine Eem-Dreiteilung durch SEIDENKRANTZ \& KNuDSEN (1997) im Kattegat und die palynologisch-terrestrische in Frankreich durch THOuveny et al. (1994) erhielt.

Deutlich sichtbare Lösseinwehung in den Intervallen zwischen dem Rocourt-, dem Pescher und dem Holzer Boden in den Profilen des Rhein- 


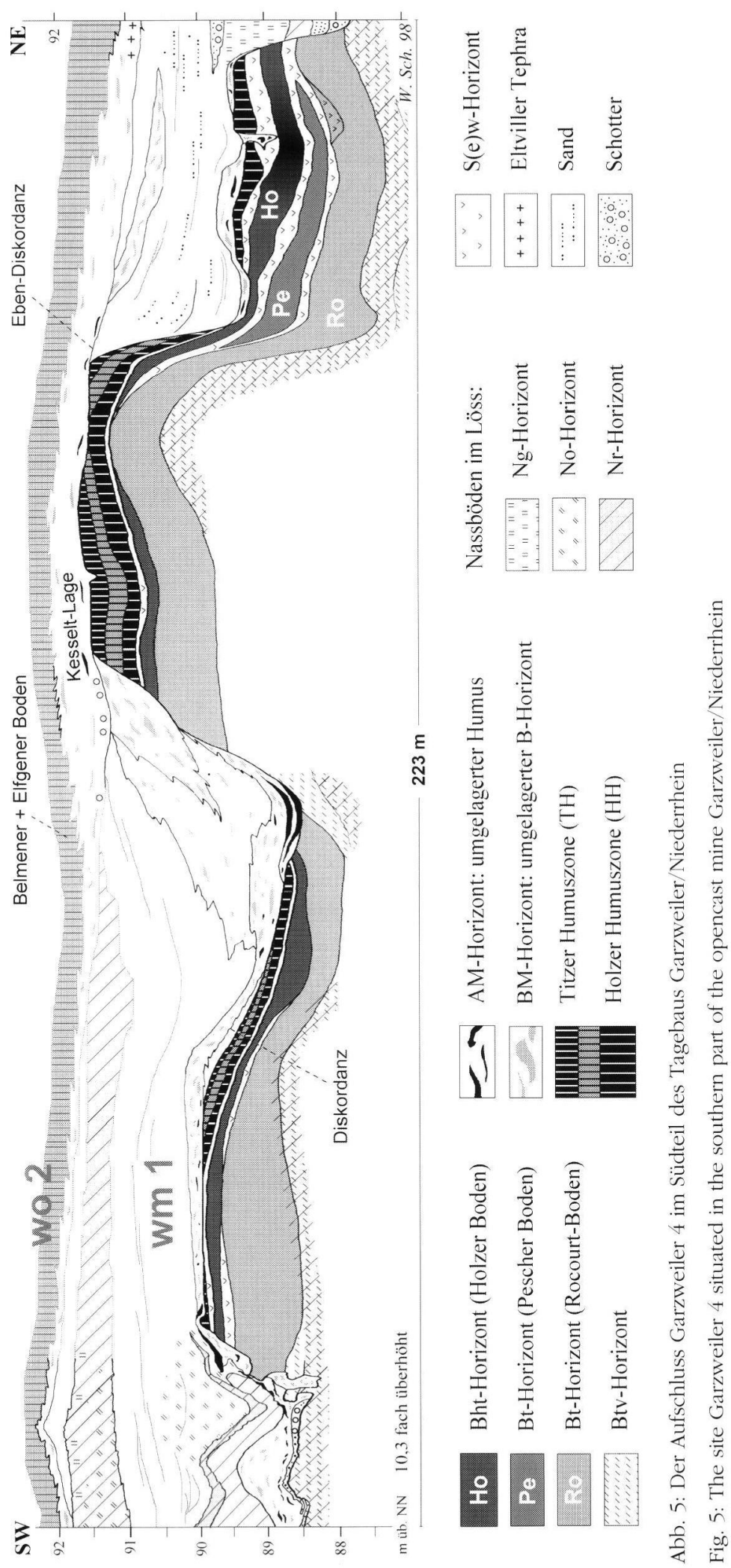

Maas-Gebietes lässt nur den Vergleich mit trennenden Kaltphasen von der Größenordnung Herning/Melisey I/Saulgau einerseits und Rederstall/Melisey II andererseits zu, wie sie eben nur über dem Eem im Unterwürm auftreten ${ }^{16}$. Derartige kurze Kaltphasen oder Breviglaziale (SCHIRMER 1999a) sind im periglazialen Lössgebiet durch geringe Sedimentaufhöhung und geringe Morphodynamik gekennzeichnet, im Gegensatz zu den Euglazialen, die kräftige Abund Umlagerung und damit weiträumige Landschaftsumgestaltung mit sich bringen.

\subsection{Mittelwürm - unge- ahnter Bodenreichtum}

Das Profil des Schwalbenbergs bei Remagen in der Goldenen Meile im Bereich der Ahrmündung in den Rhein (Abb. 2a und 7) erschließt neun braune Interstadialböden und dazu eine Reihe von Nassböden, die alle deutlich von Lössablagerungen getrennt sind (W. SCHIRMER 1990, 1991, 1995a17). Die braunen Böden sind vom Typ der Kalkbraunerde. Sie sind jeweils durch erhöhte C-Gehalte und erhöhte Tongehalte gekennzeichnet. Ihre Molluskenfauna zeigt reichere Individuenzahl, Artenzahl und wärmere Ansprüche (Schiermeyer 2000).

Der tiefste der braunen Böden, der Reisberg-Boden (Abb. 3), liegt in einem mächtigen $\mathrm{Ab}-$ schnitt von Lössfließerden und ist damit deutlich von den jüngeren, höher im Mittelwürm gelegenen Böden abgesetzt. Die acht braunen Böden darüber gruppieren sich in eine Zweiergruppe, die Unteren Remagener Böden (R1-2) und eine Dreiergruppe, die Oberen Remagener Böden (R3-5) und - 
getrennt durch ein etwas mächtigeres Lösspaket (Kripp-Stadial) - eine weitere Dreiergruppe, die Sinziger Böden 1-3. Fasst man die Remagener Böden als eine Bodengruppe zusammen, so ergeben Ausbildung und Anordnung der braunen Böden und zwischenliegenden Lösse eine Vierteilung des Mittelwürms (wm 1-4): wm 1: mächtiger Löss mit Reisberg-Boden - wm 2: mächtige Interstadialgruppe der Remagener Böden 1-5 - wm 3: schmaler Löss (Kripp-Stadial) - wm 4: schmale Interstadialgruppe der Sinziger Böden 1-3. Die Fünfergruppe der Remagener Böden zeigt eine weitere Unterteilung in eine Zweiergruppe (Untere Remagener Böden: Remagen 1-2) und in eine Dreiergruppe (Obere Remagener Böden: Remagen 3-5) mit dazwischen liegendem etwas breiteren Löss als zwischen den übrigen Remagener Böden. Auffallend und wichtig für den späteren Vergleich ist ferner, dass der Boden Remagen 2 der dickste von den Unteren Remagener Böden ist, der Boden Remagen 3 der kräftigste von den Oberen Remagener Böden ist und der Boden Sinzig 1 den kräftigsten unter den Sinziger Böden stellt.

Die besten Vergleichsmöglichkeiten zum Schwalbenberg-Profil finden sich wiederum in den Kurven der grönländischen Eiskerne und solchen aus Tiefseebohrungen. Abb. 9 und 10 zeigen den Vergleich der Schwalbenberg-Bodenfolge (Remagener und Sinziger Böden) mit dem GRIP-Summit-Eiskern (DANSGAARD et al. 1993), Abb. 9 zusätzlich den mit der Tiefseesedimentkurve DSDP609 aus dem Nordatlantik (BOND et al. 1993) und der Tiefseesedimentkurve KET 8004 aus dem Tyrrhenischen Meer (PATERne et al. 1986). Aufschlussreich ist auch der $\mathrm{C}_{\text {org }}$-Gehalt des Schwalbenberg-Profils in Abb. 7.

Der Vergleich zeigt folgende Gemeinsamkeiten: MIS 4 ist überall als breite Kaltphase entwickelt. Es folgen bis zur MIS 2-Kaltphase des Oberwürms drei Warmphasen-Gruppen (Interstadial-Gruppen) getrennt durch etwas tiefere, aber kurze kalte Einschnitte. Sie sind in Abb. 9 als MIS 3e, $3 c$ und 3 a bezeichnet. Die Interstadial-Gruppe 3e, die der Unteren Remagener Böden, umfasst zwei Wärmegipfel, R1-2 der Rhein-Lössfolge, IS 17-14 in Summit, ein kleinerer und größerer Gipfel in DSDP-609 und 3.37 und 3.35 in KET 8004. Hierbei ist jeweils der zweite Gipfel der kräftiger ausgeprägte. Nach kurzem Kälterückschlag folgen im MIS 3c drei Wärmegipfel: Obere Remagener Böden R 3-5, IS 12-10 in Summit und DSDP-609, 3.33-3.31-3.13 in KET 8004. Von ihnen ist in den meisten Kurven der erste der kräftigste; in KET
8004 konkurrieren die ersten beiden um die Vorherrschaft. Der dritte ist der schwächste. Es folgt ein sehr ausgeprägter Kälteeinschnitt, gefolgt von einer Dreier- bis Vierergruppe von Warmgipfeln in MIS 3a: Sinziger Böden S 1-3, IS 8-5, 3.1-3.033.01. Bei allen ist der älteste Gipfel der weitaus stärkste. Die folgenden jüngeren Peaks werden immer schwächer. Das ist wohl auch der Grund, weshalb sich die letzten beiden wechselnd als zwei oder nur als ein Gipfel abbilden.

Die hier vorgetragenen Korrelationen sind kein Einzelfall. Nahezu alle Kerne, die das Mittelwürm durchfahren, lassen diese genannten Merkmale erkennen. Ein Vergleich des Mittelwürms vom Schwalbenberg mit den grönländischen Bohrkernen Dye 3 und Camp Century ist in W. SCHIRMer (1995a: 531) vorgeführt. Ähnlich guten Vergleich lässt eine Staubdichtekurve aus dem grönländischen Eiskern Dye 3 (Hammer et al. 1985) zu. Der antarktische Vostok-Eiskern (Jouzel et al. 1987) lässt zumindest die Dreiteilung des Mittelwürms 2-4: warme Remagen-Gruppe - kaltes Kripp - warme Sinzig-Gruppe erkennen.

Solche bis in kleinste Einzelheiten gehende Gemeinsamkeit wohlfestgelegter Klimaschwankungen sollte alle Zweifel an der Gültigkeit der Korrelation beiseite räumen. Prähistorische, Radiokarbon- und TL-Alter unterstützen diese Korrelation der Klima-Verhaltensmuster aufs Beste: Vom Oberteil der Sinziger Böden aus nächster Umgebung stammt ein Artefaktinventar, das typologisch dem Übergang Mittel- bis Jungpaläolithikum zugeschrieben wird. Aus dem Artefakthorizont stammen ${ }^{14} \mathrm{C}$-Daten von Mollusken mit Altern von $27.890 \pm 440$ (Pta-2722) und $28.080 \pm 530$ (Pta-2721) (App et al. 1987: 100). Eine TL-Datenserie aus 44 Proben über die Remagener und Sinziger Böden hinweg verteilt ergab Werte zwischen grob 50.000 und 30.000 a (Altersbestimmung M. FreChen)18; dabei ist bekannt, dass TLAlter ins tiefere Würm hinein sich zunehmend als etwas zu jung erweisen.

In Mitteleuropa gibt es keine Mittelwürm-Folge, die eine ähnlich starke Differenzierung wie diejenige des Schwalbenbergs aufweist. Die nächste beste Vergleichsmöglichkeit bietet das Pollenprofil von der Grande Pile in den Vogesen (WoIlLARD \& Mook 1982, De Beaulieu \& Reille 1992), das in Abb. 7 mit dem Schwalbenberg-Profil korreliert ist.

\subsection{Oberwürm - Löss mit Lücken}

Der Oberwürmlöss des Rhein-Maas-Gebietes zeigt eine auffallende Zweiteilung (Abb. 3). Der 
0
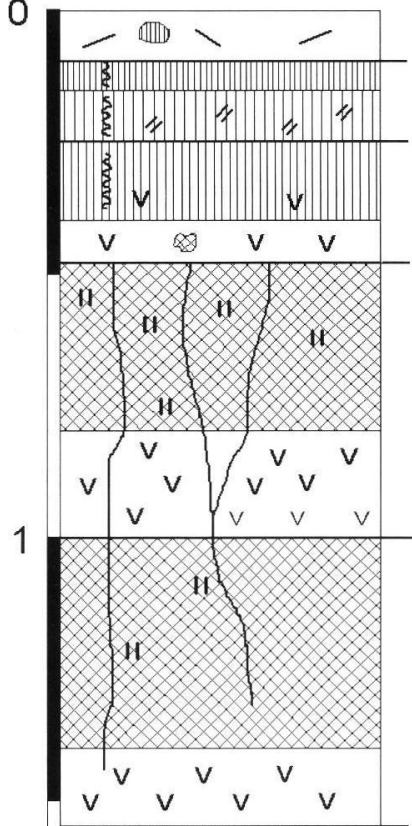

11

in

ii

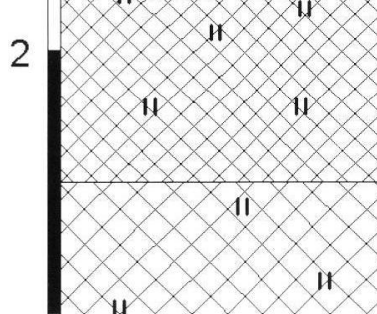

II

iI

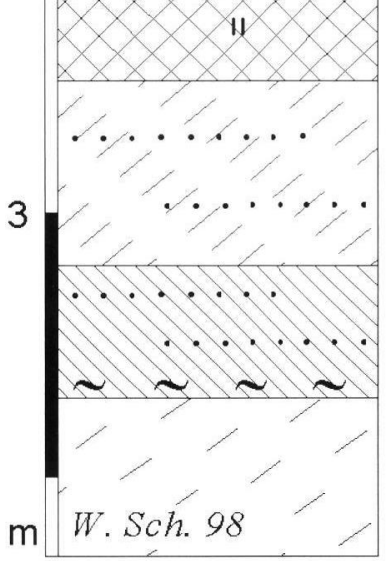

BMAh2Sew

fSdBht

SdBhtSw

BtSw

SdBhfBt

AlSwfAh1

SdBhtSW

fSdBt1

SdBt2

$\mathrm{SdBtNr}$

$\mathrm{Bt}+\mathrm{Bbv}$

BtBbv1Nor

Bbv2
AMCV

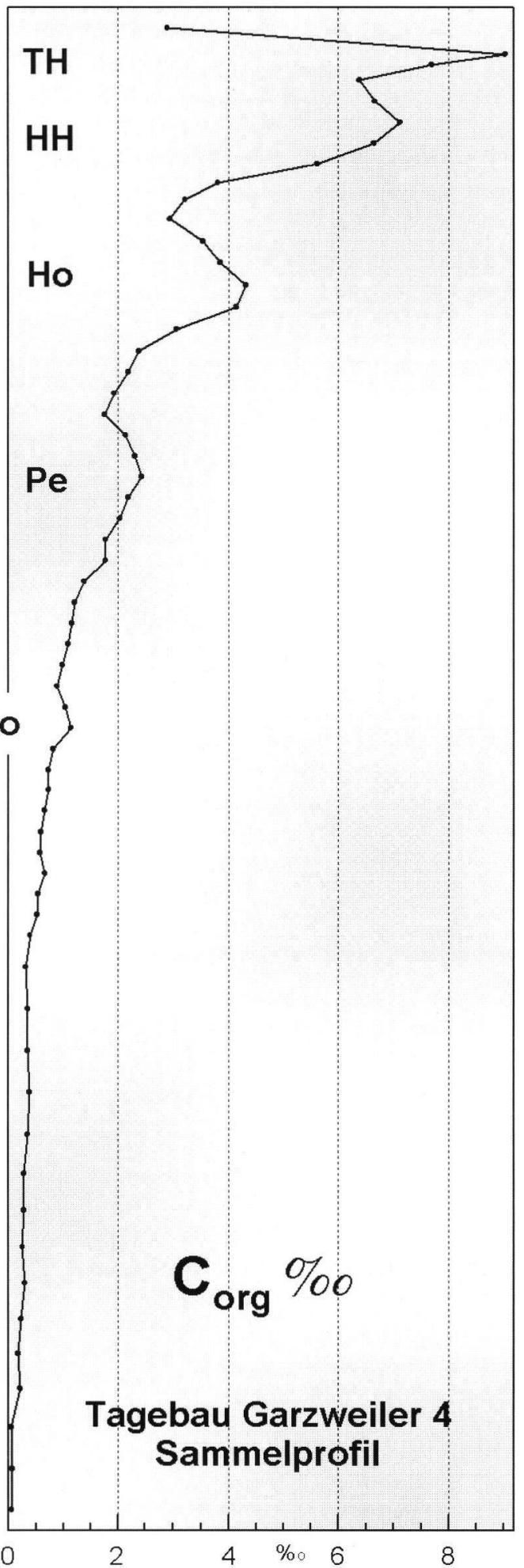

Abb. 6: Sammelprofil Garzweiler 4 samt $C_{\text {Org }}$-Gehalt. Legende siehe Abb. 4.

Fig. 6: Compiled section Garzweiler 4 with $\mathrm{C}_{\text {org }}$ content. Legend see Fig. 4. 
untere Teil, der Hesbaye-Löss, ist häufig feingeschichtet (niveo-äolischer Löss der belgischen Kollegen 19 ) und durch kräftige Nassböden (Erbenheimer Böden 1-3) gegliedert. In seinem obersten Teil stellt die Eltviller Tephra als einige mm dickes, schwarzes Band den besten Leithorizont im letztglazialen Löss des Rhein-Maasgebietes dar (vgl. Meijs et al. 1983). Örtlich unterschiedlich, von noch zeitlich vor bis zeitlich nach der Ablagerung dieser Tephra, setzt eine weiträumige flächenhafte und lineare Erosion ein, die durch die Eben-Diskordanz an der Basis der Kesselt-Lage sichtbar wird (W. SCHIRMEr 2000b). Diese Erosion arbeitet große Teile älteren Lösses auf, lagert dabei viel entkalktes Löss- und Bodenmaterial einschließlich Sand und Geröll um (KesseltLage). In dieser Zeit der Umlagerung setzt die Lösseinwehung aus, erkennbar an fehlendem Kalkgehalt in Bereichen umgelagerter entkalkter älterer Lösse.

Auf diesen längeren Stillstand setzt die Lösseinwehung erneut ein und leitet damit die letzte große Lösssedimentationsphase im Würm ein, die den frischen, hellen, sehr homogenen obersten Löss als ziemlich gleichmäßige äolische Decke absetzt, den Decklöss im Sinne WunsTORF (1913: 319) oder Brabant-Löss im Sinne Gullentops (1954).

Dieser Brabant-Löss wird durch zwei kräftigere Bodenbildungsphasen unterbrochen (Abb. 3). Die erste setzt kurz nach Ablagerung der ersten Dezimeter Lösses ein. Auf einen sehr schwach humosen Nassboden (Belmener Boden) folgt ein kräftig humoser Boden vom Pararendzina-Typ (Elfgener Boden), der lokal mehr oder weniger vernässt ist. Da der hellgraue Belmener und der humosbraune Elfgener Boden vielerorts innig solifluktiv mit der liegenden gelb- bis rötlichorange getönten Kesselt-Lage vermengt sind, stellen sie einen auffallenden Leithorizont in vielen Aufschlusswänden des Rhein-Maas-Gebietes dar. Daher habe ich diesen Leithorizont von der Basis der Kesselt-Lage bis zur höchsten Vernässung in oder knapp über dem Elfgener Boden mit einem sämtliche Einzelglieder zusammenfassenden $\mathrm{Na}$ men belegt, die Eben-Zone (W. Schirmer 2000b). Sie umgreift die Grenze der beiden großen Lösseinheiten des Oberwürms, des Hesbaye- und Brabant-Lösses20.

Einzelglieder dieser Abfolge werden auch in Abb. $5 \mathrm{im}$ Aufschluss Garzweiler 4 sichtbar (W. SCHIRMER 1999b). Dort folgt über dem Rocourt-Solkomplex tieferer Ahrgau-Löss (wm1), der vor allem - und das ist typisch für ihn - aus mächtiger Lössfließerde besteht, die das liegende Bodenmaterial mit aufgearbeitet hat. Sie enthält zwei Nassböden, die noch nicht im Schema der Abb. 3 verzeichnet sind, da ihr Auftreten noch nicht als regelhaft erwiesen ist. Die Folge wird wiederum durch die im Niederrhein-Maas-Gebiet weit und flächenhaft verbreitete Eben-Diskordanz geschnitten, deren Aufarbeitungsergebnis die Kesselt-Lage verkörpert. Basal mit kleinen Frostspalten und kleinen Geröllen enthält die Kesselt-Lage ansonsten viel umgelagertes Bodenmaterial und Fetzen der Eltviller Tephra. Darüber folgt der charakteristisch geschwänzte hellgraue Belmener und ebenfalls geschwänzte, dunkelgraue, humose Elfgener Boden (in Abb. 5 als ein gemeinsamer Boden dargestellt). Mit diesen beiden Böden beginnt der BrabantLöss des höheren Oberwürms (wo 2). Kesselt-Lage, Belmener und Elfgener Boden bilden gemeinsam die Eben-Zone.

Im höheren Brabant-Löss tritt des Weiteren ein brauner Boden auf, der Leonard-Boden. Er ist manchmal zweigeteilt und inmitten, wie auch in seinem Liegenden und Hangenden von Nassböden begleitet. Es ist ein Kalkbraunerde-Typ, wie er sonst so zahlreich im Mittelwürm auftritt. Er ist an Tagebauwänden oft kilometerweit erschlossen, allerdings gelegentlich durch den Durchgriff der heutigen Parabraunerde der Oberfläche nach unten maskiert. Über dem Leonard-Boden folgt noch ein kräftiger Nassboden (Gustorfer Boden), der besonders durch Füllungen tiefreichender Eiskeile, die die Oberflächenbodenbildung nicht überdeckt hat, hervortritt 21 .

Während im Niederrhein-Maas-Gebiet der Brabant-Löss als Decklöss oberflächlich am häufigsten - jedoch nicht allerorts - ausgebildet ist, ist durch die Erosionstätigkeit an der Eben-Diskordanz der Hesbaye-Löss nur in Schutzpositionen des Reliefs, also in Hohlformen oder in Leeposition erhalten. Auf das Konto der Eben-Diskordanz und auch älterer Abtragungs-Diskordanzen geht auch die starke Dezimierung des Ahrgau- und Rheingau-Lösses im Niederrhein-Maas-Gebiet, die mancherorts unter dem Brabant-Löss nicht erhalten sind.

Einstufung: Die Eltviller Tephra ist die beste Leitlage im Profil. Ihre ${ }^{14} \mathrm{C}$ - und TL-Datierung hat nach Frechen \& Preusser (1996) die erhebliche Datenspannweite zwischen 31 und $16 \mathrm{ka} \mathrm{v}$. h. ergeben, mit Schwerpunkt um 20 ka. Die Tephra liegt knapp unter bis im Bereich der Kesselt-Lage. Nach sedimentologischen Überlegungen sollte das Letztglaziale Maximum (LGM) im oberen Hesbaye-Löss, in der Kesselt-Lage, liegen. Die Kesselt-Lage ist eine großregionale Permafrostauftaulage und sollte Auslöser für das letzte und weiteste Auseinandergleiten der oberwürmzeitlichen Eiskalotte gewesen sein (W. SCHIRMER 2000b). 


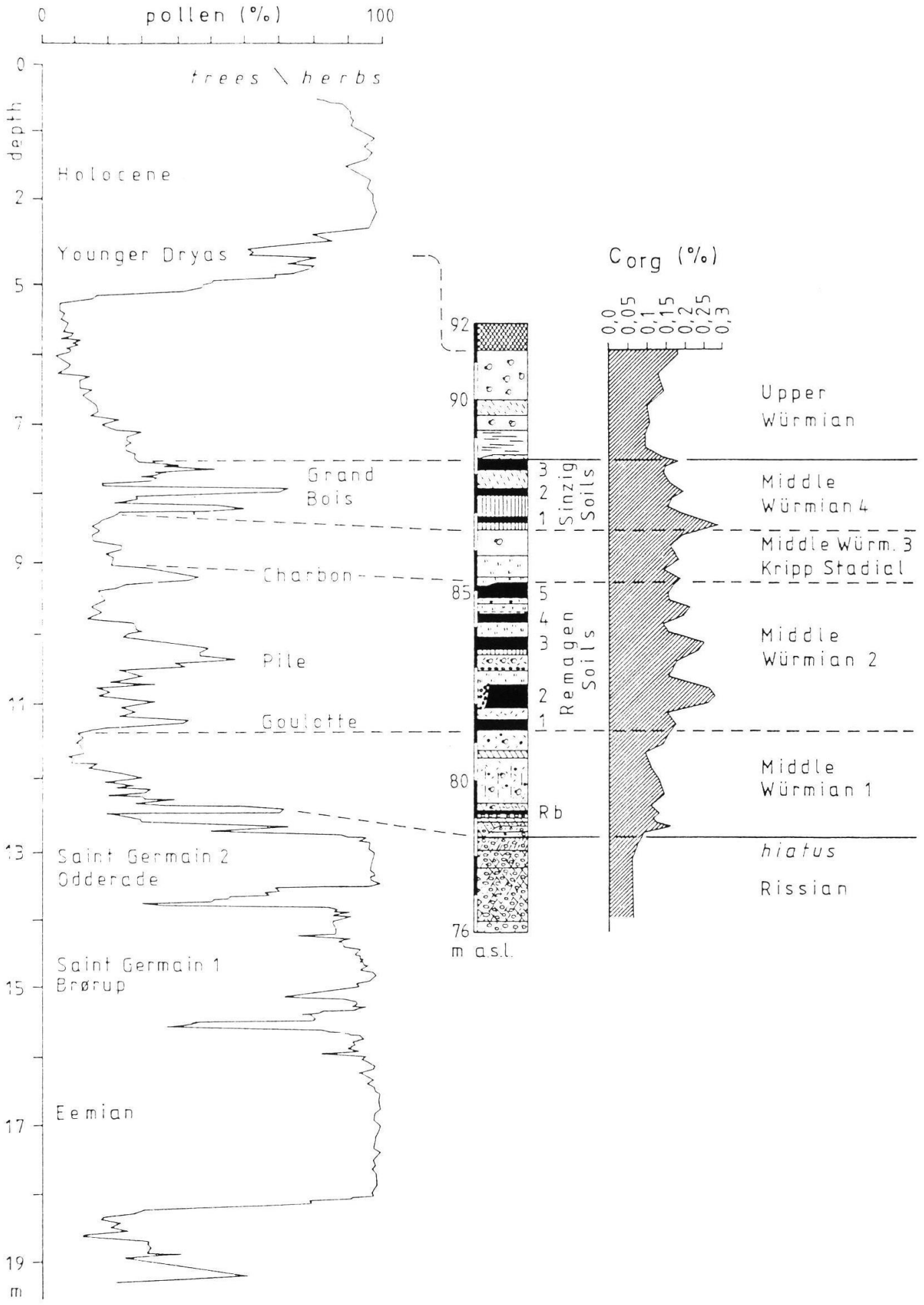

Abb. 7: Grande Pile-Profil: Baum- und Strauchpollen gegen Kräuterpollen nach WoILLARD \& MOOK (1982: 159) verglichen mit dem Schwalbenberg-Profil (aus W. ScHIRMER 1995c: 513; wenig ergänzt)

Fig 7: Grande Pile section: total tree + shrub pollen versus herb pollen from WOILLARD \& MoOK (1982: 159), correlated with the Schwalbenberg section (after W. SCHIRMER 1995c: 513; slightly modified) 
Wenig später setzt die Warmphase des Elfgener Bodens ein 22 . Sie dürfte den Eisrückzug eingeleitet haben. Das Zusammenbrechen des Permafrostbodens sollte es auch sein, das in den Talböden den Umbruch von der Maxiwürm-Terrasse zur Schönbrunner Terrasse verursacht hat. Abtrag an der Eben-Diskordanz unter Lieferung großer Siltmengen, folgende Erwärmung zur Zeit des Belmener und Elfgener Bodens sollten Konzentration des hochkaltzeitlichen Breitbettflusses zum schmalen Mäandrieren und Eintiefen angeregt haben und einen Flusstyp mit Auendynamik mit reicher Siltversorgung geschaffen haben, wie er in der Schönbrunner Terrasse verwirklicht ist. Zur Zeit des folgenden Brabant-Lösses mit erneut einsetzenden Permafrost-Bedingungen griffen dann die Flüsse in der Schönbrunn-Phase als Breitbettflüsse über fast das ganze Tal wieder aus, von der Maxiwürmterrasse allerdings durch eine deutliche Terrassenkante abgesetzt (W. SCHIRMER, zuletzt 1995d).

\section{Spätwürm}

Mit dem Spätwürm ist die wesentliche Lössbildung abgeschlossen, denn es setzt wieder Bodenbildung ein, die spätwürmzeitliche Bodenbildung, die schließlich in ebener Lage den ersten Ansatz der heutigen holozänen Bodenbildung darstellt. Im Norden des Lössgebietes schaltet sich seit dem höheren Oberwürm mehr und mehr Flugsand in den Löss ein, der dann in den kühleren Phasen des Spätwürms dominiert und spätwürmzeitliche Bodenbildungsphasen konserviert, wie den Usselo-Horizont. Im Mittelrheingebiet, im Bereich der Verbreitung der spätallerødzeitlichen Laacher See-Tephra (ca. 13.000 Dendrojahre vor heute oder 11.000 Radiokarbonjahre BP) hat sich im Schutze der Tephra die Bodenbildung des Spätglazials erhalten, der Mendiger Boden 23 . Wie Untersuchungen in MiesenheimAuf der Holl" zeigen (U. Schirmer 1995, 1996 und W. Schirmer 1996) setzt die Bildung des Mendiger Bodens, die zu einer Pararendzina auf dem Brabant-Löss führt, mit dem Meiendorf-Interstadial ein, setzt sich im Bølling fort und wird im späten Allerød durch die Laacher See-Eruption abgebrochen und konserviert.

\section{Folgerungen}

4.1 Der oben durchgeführte regionale Vergleich des rheinischen Eem-Unterwürms baut auf der Orientierung am basalen Eem-Alter und der relativen klimatischen Wertigkeit der einzelnen Horizonte auf, dem Wärmephasen-Schema: eine sehr kräftige - zwei kräftige - eine Gruppe schwacher, aber scharf abgegrenzter Wärmephasen. Darüber hinaus stützt er sich auf Sedimentationsraten und Diskordanzen. ${ }^{14} \mathrm{C}$-Datierung ist in diesem Bereich nicht mehr zuverlässig, da sie die äußerste Grenze ihrer Auswertungsfähigkeit erreicht oder überschreitet. TL-Datierung erscheint für Feinstratigraphie im Unterwürm auch zu grob und kann an Bohrungen meist nicht angewandt werden. Die mit dem Warmphasen-Schema durchgeführte Korrelation ist ein Versuch, der allerdings bestechende Übereinstimmung aufzeigt. Erweist sie sich als richtig, dann löst sie wesentliche Zuordnungsprobleme mitteleuropäischer Quartärstratigraphie.

Einmal bot sie eine Lösung für den lange geäußerten Widerspruch zweier biostratigraphischer Interstadiale (Brørup/Odderade) gegenüber drei Humuszonen im Rheingau und im östlichen Mitteleuropa an. Zum andern wirft sie neues Licht auf die Frage der Stellung der Interstadiale Oere und Glinde, die infolge ihrer ${ }^{14} \mathrm{C}$-Alter einerseits im Mittelwürm 2 angesiedelt wurden, andererseits aber im Liegenden die mächtige Kaltphase des Mittelwürms 1 (MWG 1) nicht erkennen lassen (vgl. die Würmklimakurve Abb. 1 von BEHRF \& LaDE und Abb. 8 des vorliegenden Textes). Zum dritten bekräftigt sie die nicht unumstrittene Meinung Vieler ${ }^{24}$, dass die St.-Germain-Warmzeiten der Grande Pile das südwestmitteleuropäische Äquivalent zu Brørup und Odderade im nördlichen Mitteleuropa darstellen. Das WarmphasenSchema zeigt eine so typische stets wiederkehrende Bodenfolge, dass kein Platz zwischen Eem und Brørup zum Einbau zweier weiterer zu suchender und nicht auffindbarer St.-GermainWarmzeiten bleibt.

4.2 Die Lokalität Garzweiler 4 eröffnet, dass die Parabraunerde des letztinterglazialen Bodens, wie sie uns im Normalfall im Gelände begegnet, eigentlich aus drei ineinander projizierten, eigenständigen Parabraunerden, bzw. deren Bt-Horizonten, besteht (Rocourt-, Pescher und Holzer Boden). Sie beherbergt also zeitlich Bodenbildungsergebnisse des Eem-Interglazials wie auch großenteils des Unterwürms. Oder anders betrachtet: Im Bereich dieses Bodens verharrte die Landschaftsoberfläche über den Zeitraum des Eems und auch des Unterwürms in annähernd demselben Niveau. Nach unseren heutigen Vorstellungen ist das etwa die Zeit von 127.00074.000 a vor heute, also rund 50.000 Jahre. Man könnte dieser Zeit noch den folgenden Abschnitt 


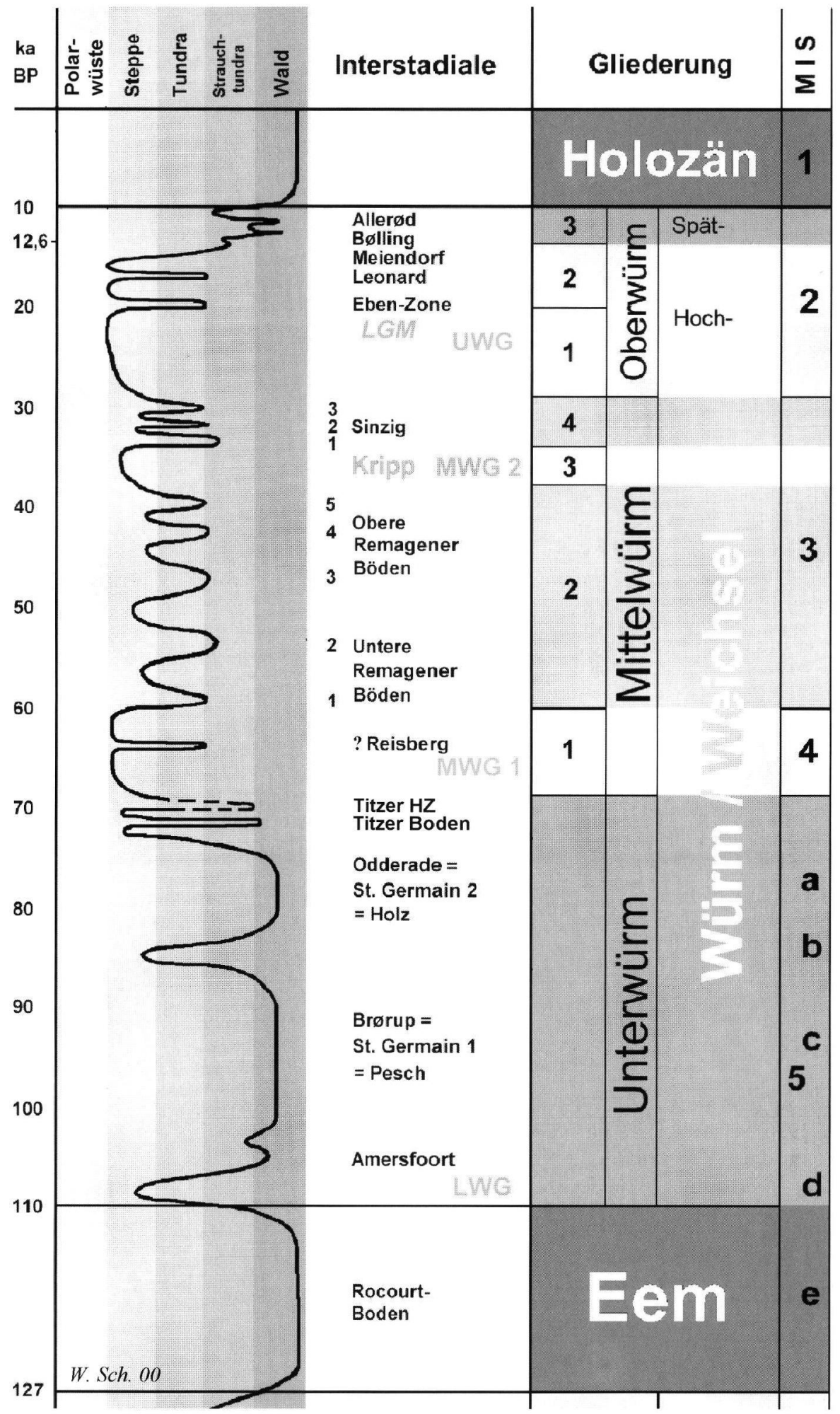

Abb. 8: Klimakurve der Letzten Eiszeit

Fig. 8: Climate curve of the Last Glacial 


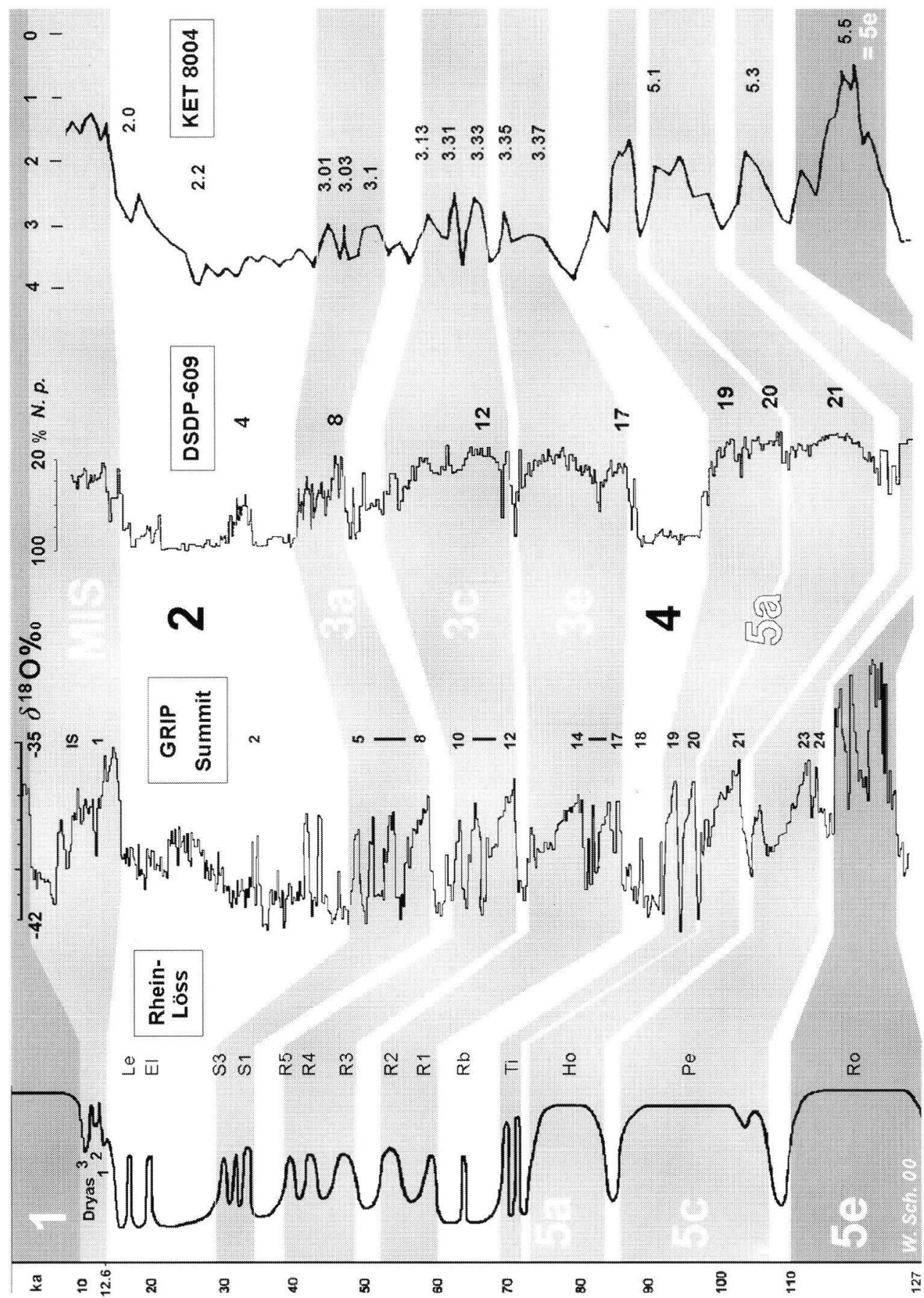

Abb. 9: Vergleich der oberpleistozänen Rhein-Löss-Bodenfolge mit dem Summit-Eiskern, dem nordatlantischen Tiefseesedimentkern DSDP-609 (BOND et al. 1993) und dem tyrrhenischen Tiefseesedimentkern KET 8004 (PATERNE et al. 1986). DSDP-609 zeigt die Häufigkeit der planktonischen Foraminifere Neogloboquadrina pachyderma, KET 8004 zeigt die 218 O-Kurve von Schalen der Globigerina bullö̈des. Abkürzungen sind in Abb. 3 erläutert.

Fig. 9: Correlation of the Rhein loess-soil sequence, the Summit ice core, the North Atlantic deep-sea sediment core DSDP-609 (BOND et al. 1993) and the deep-sea sediment core of KET 8004 in the Tyrrhenian Sea (PATERNE et al. 1986). DSDP-609 shows the abundance of the planktic foraminifera Neogloboquadrina pachyderma, KET 8004 the $18 \mathrm{O}$ record of Globigerina bulloïdes. For abbreviations see Fig. 3. 


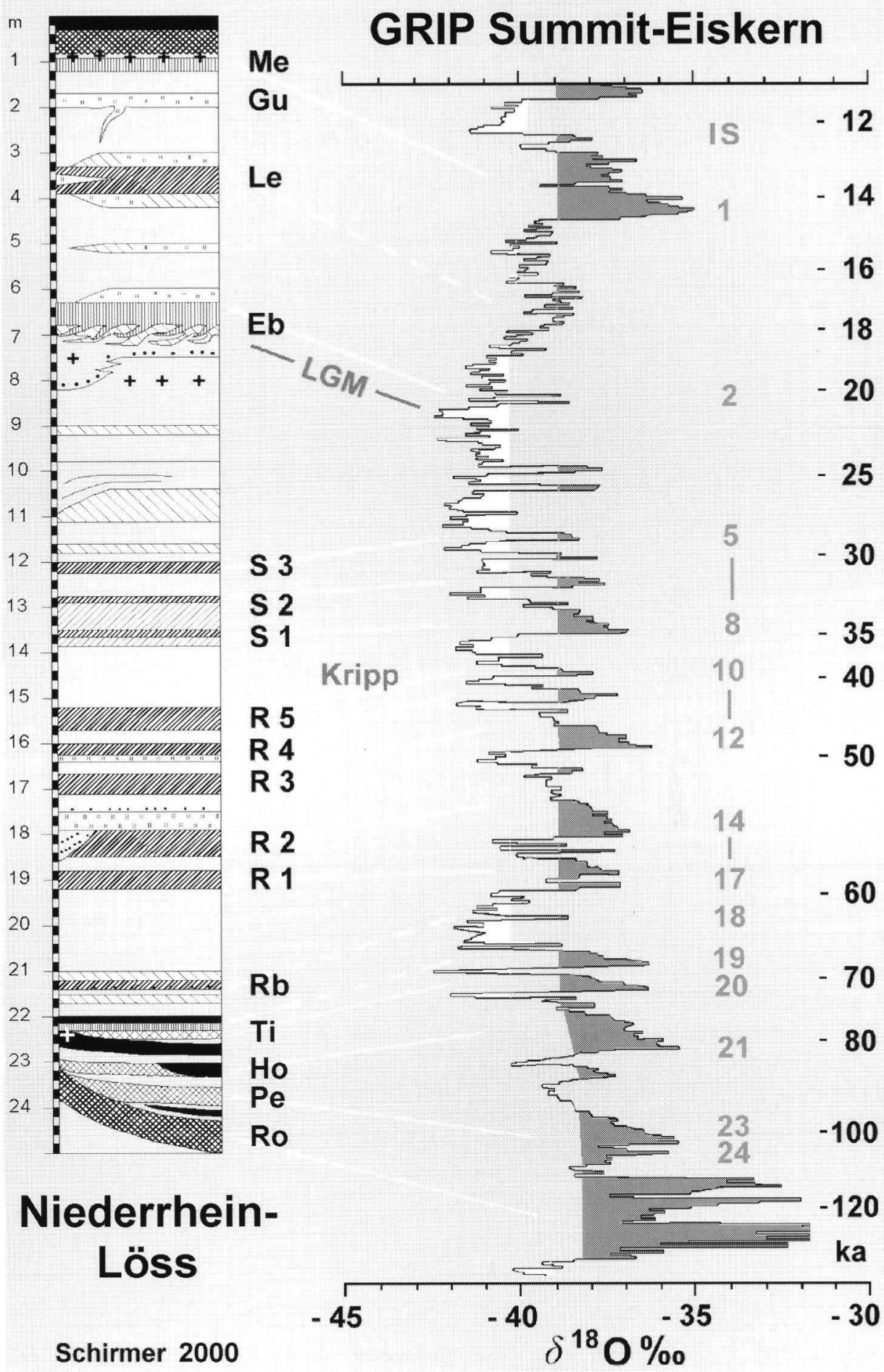

Abb. 10: Vergleich der oberpleistozänen Rheinlöss-Folge mit dem grönländischen Summit-Eiskern (DANSGAARD et al. 1993).

Fig. 10: Correlation of the Late Pleistocene Rhein loess record with the Greenland Summit ice core (DANSGAARD et al. 1993). 
der Holzer Humuszone, des Titzer Bodens und der Titzer Humuszone hinzufügen, in dem sich abgesehen von ein paar Dezimetern Aufhöhung und geringer örtlicher Erosion keine wesentliche Landschaftsveränderung zutrug. Dann betrüge die Zeit landschaftlicher Stabilität von 127.000 bis 67.000 a v. h., also rund 60.000 Jahre. Knapp noch einmal so lange währt dann die folgende Letzte Kaltzeit - einschließlich deren Interstadiale -, in der die Landschaft mehrere Male wesentlich umgestaltet wurde.

Der Rocourt-Solkomplex ist also Ausdruck einer rund 60.000 Jahre langen stabilen Periode. Vom landschaftsgeschichtlichen Standpunkt her würde man dem Erdgeschehen gerechter werden, fasste man den gesamten Rocourt-Solkomplex, entsprechend dem MIS 5, als Interglazialkomplex auf 25.

Natürlich gab es in den kurzen, sehr kalten Lücken nach dem interglazialen Höhepunkt, dem Eem, wie auch nach dem Brørup, dem Odderade und dem Titzer Boden, vorübergehend kaltzeitliche Bedingungen (Breviglaziale). Während derer gab es örtlich eine gewisse Sedimentation, geringe Lösseinwehung, aber auch geringen Abtrag. Letzterer äußert sich unter anderem darin, dass die Einzelglieder des Rocourt-Solkomplexes meist bis in den Bt-Horizont hinein durch Abtragung erniedrigt sind. So kann man den Standpunkt vertreten, mit dem Ende des Rocourt-Bodens, Ende des Eems, setzt die neue Kaltzeit, wenn auch nur als Kurzbesuch, ein, im Alpenvorland vermutlich mit kurzem kräftigem Gletschervorstoß (Welten 1981: 190, Frenzel 1991: 398, ElLWANGER 1995: 508), desgleichen vielleicht auch in Skandinavien (MANGERUd 1991: 314). In Abb. 8 ist er mit LWG, Lower Würmian/Weichselian Glaciation, bezeichnet. Nach Welten (1981: 200) war es palynologisch der kälteste Abschnitt im Unterwürm. Die folgenden Warmphasen (Brørup, Odderade) haben wohl interglazialähnliche Verhältnisse erreicht (daher St.-Germain-Interglaziale bei WOILLARD 1978: 15), bleiben aber bei starkem Vegetationsgefälle nach Norden hin (GRÜGER 1979: 32) doch knapp unter unserem heutigen Klima blieben also per definitionem Interstadiale. Vom Definitionsbegriff her beginnt somit die letzte Kaltzeit mit dem Eem-Ende, dem Herning-Stadial (s. Anm. 15). Von der Landschaftsgeschichte her betrachtet, beginnt sie erst mit dem Ende des gesamten Rocourt-Solkomplexes, rund 45.000 Jahre später.

Im Falle des Prä-Eems werden alle WarmzeitKomplexe, deren Einzel-Warmphasen durch Breviglaziale getrennt sind, zu Interglazialkomple- xen zusammengefasst - so auch bezüglich der Bodenkomplexe, deren Einzel-Bt-Horizonte durch kurze breviglaziale Lössphasen unterbrochen sind. Der Rocourt-Solkomplex wäre also der einzige Warmphasenkomplex, dessen Einzelglieder auf ein Interglazial (Eem) und das folgende Frühglazial (Unterwürm) aufgeteilt werden.

4.3 Die Lokalität Schwalbenberg ist ob ihres wohlgespreizten Mittelwürms faszinierend, aber stellt reiche Aufgaben: Die meisten Mittelwürmlokalitäten zeigen anstatt der acht Remagener und Sinziger Böden nur einen, zwei oder drei Böden (siehe Kap. 1). Hier ist zu entscheiden, welcher dieser Einzelböden mit welchem des Schwalbenbergs korreliert werden kann. Ist die Minderzahl andernorts durch Abtragungsdiskordanzen verursacht und wo liegen die Diskordanzen dann stratigraphisch? Oder sind mehrere Böden mangels Sedimentation zu einem kondensiert? Diese offene Frage ist der Grund, weshalb die Schwalbenberg-Bodenfolge nicht mit Begriffen wie Lohner Boden (Schönhals et al. 1964) oder Böckinger Boden (Bibus 1989: 8) in Zusammenhang gebracht werden kann. Als Lösungsansätze bieten sich an: Individualcharakteristik einzelner Böden; Nutzung dieses optimalen Bereiches der TL-Datierung; Suche nach dem paläomagnetischen Laschamp-Event als Leithorizont.

4.4 Das höhere Mittelwürm, eingebettet in die Kaltphasen der Mittelwürm 1-Vergletscherung (MWG 1) und Oberwürm-Vergletscherung (UWG), hatte durch seinen wohlbekannten wärmeren Gesamtcharakter, aber vage stratigraphische Festlegung der bisher gefundenen EinzelWarmschwankungen, eine rege Diskussion ausgelöst. Die Torfinterstadiale Moershoofd, Hengelo und Denekamp sind als räumlich isolierte Vorkommen definiert. Es gibt kein Profil, in dem sie in echtem Übereinander auftreten. Ihre stratigraphische Altersanordnung beruht auf ihren ${ }^{14} \mathrm{C}$ Altern.

Zusätzlich betont VANDENBERGHe (1985: 33, 35), dass Torfe nicht zwangsläufig interstadial sein müssen, sie vielmehr an feuchte geomorphologische Positionen, auch kalter Klimate, gebunden sind. Auch Behre (1989: 41) weist darauf hin, dass während der milden Phase im höheren Mittelwürm edaphisch bedingt hier und dort einmal Humus oder Torf gebildet werden konnte und dass man andererseits versucht, ${ }^{14} \mathrm{C}$-datierte Mittelwürm-Bildungen allein auf Grund ihrer Radiokohlenstoff-Alter den niederländischen Interstadialen zuzuordnen, dass schließlich im Mittel- 
würm Europas ausgeprägte Erwärmungs-Phasen fehlen, die sich über weitere Strecken korrelieren ließen.

Die Rheinlöss-Folge zeigt dagegen sehr deutlich ein klares stratigraphisches Übereinander all dieser Wärmeschwankungen, sowohl der des Unterwürms, des Mittelwürms als auch des Oberwürms als auch aller dreier Gruppen untereinander. Nicht einer der in Abb. 3 dargestellten Böden stammt aus isoliertem Kontext oder ist aus irgendwelchen Überlegungen in seine gegebene stratigraphische Position eingeschoben worden. Sie alle treten in zweifelsfreiem Übereinander auf - wohl nirgends alle in einem gemeinsamen Profil, aber stets als große Ausschnitte, die ausreichende Überlappung zum nächsten Ausschnitt gewährleisten. Und noch etwas bietet die Rheinlöss-Folge: Sie zeigt deutlich, dass im höheren Mittelwürm wohldefinierte und markante Wärmeschwankungen vorliegen, die zeigen, dass sich terrestrische, marine und glaziäre Profile sehr wohl verbinden lassen und dass Klimaschwankungen über weite Räume bis in Feinheiten gleichartig abgebildet werden konnten.

4.5 Das letzte Glazial zeigt zwei klare lössreiche Höhepunkte: die Zeit der Mittelwürm-Vergletscherung 1 (MWG 1) im wm 1 entsprechend dem MIS 4, und die Zeit der Oberwürm-Vergletscherung (UWG) im wo 1/2 entsprechend dem MIS 2. Die MWG 1 war allgemein feuchter, die UWG trockener. Beide Abschnitte zeigen im Niederrhein-Maas-Raum die mächtigsten Lössablagerungen. Dennoch haben beide Höhepunkte auch ausgeprägte lössfreie Phasen und Bodenbildung, das heißt Vegetationsvermehrung: Reisberg-Boden im wm 1, Eben-Zone und Leonard-Boden im wo 226. Selbst innerhalb dieser beiden Hochglazialphasen waren also die Kältehöhepunkte, von wärmeren Phasen unterbrochen auf einzelne kürzere Zeitabschnitte eingeengt.

4.6 In Abb. 8 ist die Rheinlöss-Folge der Letzten Eiszeit in eine Klimakurve umgeformt. Sie fußt auf lithologischen und pedologischen Klimaindikatoren und solchen der Flora und Fauna. Betrachtet man das Verhältnis Lössablagerung zu Bodenbildung in der Zeit, so stellt man fest, dass den Bodenbildungsphasen im rheinischen Löss mehr Zeit zukommt als den Lösssedimentationsphasen. In jedem Falle erhält die Letzte Eiszeit durch die hier vorgetragene Vermehrung der Böden mehr Vegetationszeit als bisher angenommen. Die Rheinlöss-Folge bereichert die Lössstratigraphie des Letzten Glazials nicht nur um einige stratigra- phisch verwendbare Glieder, sie hat auch die Letzte Eiszeit im Gesamthaushalt etwas wärmer gemacht.

\section{Dank}

Der Deutschen Forschungsgemeinschaft gebührt Dank für finanzielle Unterstützung dieses Projektes. Den Herren Kollegen Jürgen EHLERs und ARMin SKOwRONEK danke ich sehr herzlich für kritische Anregungen zum Manuskript.

\section{Anmerkungen}

1) Dieser Text ist eine erweiterte Fassung eines Vortrags anlässlich der DEUQUA-Tagung in Hannover im September 1998 (W. SCHIRMER 1998). Er wurde 1998 erstellt in der Annahme, dass er 1999 gedruckt würde. Daher ist in den später geschriebenen, aber früher erschienenen Arbeiten (W. SCHIRMER 1999a, b, c) auf ihn als Quelltext verwiesen worden.

2) Zur Lage der namengebenden Lokalitäten siehe Abb. $2 a$.

3) Der vorliegende Text stellt eine Übersicht über die Ergebnisse der Oberpleistozän-Gliederung am Unteren Mittelrhein und im Niederrhein-Maas-Gebiet dar. Berichte zum Eem/Unterwürm sind in W. SCHIRмer (1999a, b), zum Mittelwürm in W. Schirmer (1990, 1991) und SChiermeyer (2000), zum Oberwürm in Henze (1998) und W. SCHIRMER (2000b) und zum Spätwürm in W. Schirmer (1996) ausgeführt. Ausführlichere Einzeldarstellungen sind in Vorbereitung.

4) Z. B. Gullentops (1954); hierin Angaben älterer Literatur.

5) Die Benennung dieser wie der Präwürm-Lösse (W. ScHIRMER 1999a) wird an anderer Stelle näher erläutert und charakterisiert.

6) In den früheren Arbeiten (W. Schirmer 1990, 1991: 73, 1992, 1995b: 541 und SCHIRMER \& FELDMANv 1992) habe ich den Gefleckten Nassboden mit NS (Nassboden vom Pseudogleytyp bzw. Haftnässepseudogleytyp) und den Grauen Nassboden mit NG (Nassboden vom Gleytyp) bezeichnet.

7) Die Existenz diachroner Nassböden wurde mir im Januar 1998 bewusst.

8) Die Namen Homburger und Erbacher Boden (SchÖNHALs et al. 1964) für den letztinterglazialen Boden in Hessen wurden 10 Jahre nach GulLENTops (1954) aufgestellt. Folgt man der Prioritätsregel, so gebührt dem Rocourt-Boden als Boden des Rhein-MaasRaumes der Vorrang.

9) Alle im Text gegebenen Abkürzungen für Namen von Böden und Leitlagen sind in Abb. 3 eingetragen.

10) Die die Humuszonen trennende Verbraunung wurde erst jüngst in gespreizter Profilposition auf wenigen Metern Länge im Profil Tagebau Garzweiler 12 am Rande eines damaligen Bachbettes beobachtet. In allen anderen Profilen des Niederrhein-Maas-Gebietes lässt die den Rocourt-Solkomplex beschließende Humuszone 
profilmorphologisch stets nur zwei humose Horizonte erkennen. Da sie in zeitlichem Zusammenhang zu den Mosbacher Humuszonen des Rheingaus stehen müssen, habe ich sie in den früheren Arbeiten (W. SCHIRMER 1999a, b, c, d, e) als Mosbacher Humuszone 1 und 2 bezeichnet. Nachdem sich aber über dem Rocourt-Bt eine eigene Humuszone fand, habe ich die Einzelglieder der den Rocourt-Solkomplex beschließenden Humuszone, da sie auf den Holzer Boden folgen, als Holzer Humuszonen (HH1 und 2) bezeichnet (W. Schirmer 2000a). Denn nahezu jeder Bt-Horizont der Rheinischen Lössfolge trägt eine Humuszone. So habe ich eine Humuszone jeweils mit dem Namen des darunter liegenden Bt-Horizontes unter dem Zusatz "Humuszone" belegt über dem Erkelenzer Boden liegt also die Erkelenzer Humuszone - dies, um die Zugehörigkeit der Humuszone sogleich erkennen zu lassen und um die Namenvielfalt nicht unnötig zu vergrößern. So bin ich dann konsequenterweise auch mit den beiden Humuszonen über dem Holzer Boden verfahren. Nachdem diese beiden Humuszonen nun eine Trennung durch einen eigenen braunen Boden verrieten, gliedere ich das Humuszonen-Paket in Holzer Humuszone, Titzer Boden und Titzer Humuszone.

11) Und so verstand es auch die genannte Subkommission. Damals übersah man noch nicht, welches Verhältnis die Humuszonen zu den Interstadialen Brørup und Odderade haben.

12) Behre \& Van der Plicht (1992: 116) sehen die Radiokohlenstoff-Daten für das Glinde-Interstadial (51.000-48.000 a BP) und Oerel-Interstadial (58.00054.000 a BP) als sicher an, währenddessen sie das Datum für die räumlich dicht darunter liegende Obergrenze Odderade (61.000 a BP) als zu jung betrachten. Mit derselben Argumentation wie für die Odderade-Obergrenze gegeben kann man sämtliche doch so dicht beieinander liegenden Daten als zu jung ansehen. Entscheidend ist meines Erachtens, dass der Beleg fehlt, dass zwischen der Obergrenze Odderade und Oerel die lange kalte Phase des terrestrischen Mittelwürms 1 (im Sinne W. SCHIRMER 1991, 1995) entsprechend dem MIS 4 im marinen Bereich vorhanden ist.

13) De Beaulieu \& Reille (1992: 433) sehen Ognon II, da es in ihrer Bohrung Grande Pile 20 fehlt und bei WolLLARD ein seltsames Pollenspektrum mit Mischung thermophiler und Steppenelemente hat, als umgelagertes Sediment an. Allerdings deutet WeLten (1981: 200) die thermophilen Anteile als Fernflugspuren, wie sie in der Westschweiz in diesem Horizont häufig auftreten.

14) Die oben verwendete Bezeichnung der FrühwürmInterstadiale orientiert sich an den Pollendiagrammen in Abb. 3-5 von Welten 1981, und denselben in Abb. 3a, b in Welten 1982. Im Text von 1981 verwendet WelteN eine andere Nummerierung seiner Frühwürm-Interstadiale.

15) Benennung als Untere, Mittlere und Obere Mosbacher Humuszone durch Semmel (1995: 134).

16) Der erste Kälterückschlag, der auf das Eem folgt und Eem von Brørup trennt, wurde von ANDERSEN (1980: 53) als Herning-Stadial bezeichnet, von WollLaRD
(1978: 16) als Melisey I, von Frenzel (1991: 398) als Saulgau-Vereisung. Der Kälterückschlag zwischen Brørup und Odderade wird als Rederstall-Stadial (MENKE 1980: 45) bzw. Melisey II (Wolllard 1978: 16) bezeichnet.

17) Die bisher vom Schwalbenberg veröffentlichten Ergebnisse stammen von einer ersten Bearbeitung des Schwalbenberg-Aufschlusses 1989. Da aus ihr die Bedeutung des Profils erst hervorging, habe ich den Aufschluss inzwischen noch einmal feinstratigraphisch beprobt und bearbeitet. Die Auswertung durch eine Arbeitsgruppe ist noch nicht ganz abgeschlossen.

18) Eine gemeinsame Publikation darüber ist in Vorbereitung.

19) vgl. GulleNtops (1954)

20) Die Eben-Zone und ihre Einzelglieder wurden besonders von belgischer und niederländischer Seite mit den verschiedensten Namen belegt, zum Beispiel enthalten sie die Begriffe Kesselt-Boden, Nagelbeek-Horizont, Horizont à langues. Eine historische Darstellung der Namen, aber auch der Verwechslungen und Missverständnisse, die um diese Begriffe entstanden, findet sich in W. SCHIRMER (2000b).

21) Das Oberwürm des südlichen Niederrheingebietes wurde auch in einer Dissertation von N. Henze (1998) bearbeitet, das des nördlichen Niederrheingebietes und der Maas ist bei W. Schirmer (2000b) dargestellt.

22) Der Belmener Boden und der Elfgener Boden samt seinen begleitenden Nassböden entsprechen zusammen nach ihrer Ausbildung und konstanten Lage über der Eltviller Tephra dem Erbenheimer Boden 4 sensu SCHÖNHALS et al. (1964).

23) Der Mendiger Boden tritt nur im Mittelrheingebiet auf. Die Laacher See-Tephra ist in der Niederrheinischen Bucht nur schwermineralogisch auffindbar.

24) Zum Beispiel Grüger (1979: 33), Welten (1981), BeHRe (1989: 35).

25) In eincm Vortrag kam der Einwurf, der Rocourt-Solkomplex könne nicht als "Interglazialkomplex" bezeichnet werden, da sich überwiegend Interstadiale daran beteiligen. Von seinem Wesen her nimmt der Rocourt-Solkomplex aber dieselbe Rolle ein wie die älteren echten Interglazialkomplexe, die tatsächlich aus mehreren definitionsgerechten Interglazialen bestehen. Interglazialkomplex bedeutet $j a$, dass sich daran auch andere Klimaabschnitte als Interglaziale beteiligen, vor allem auch Breviglaziale als kühlste Abschnitte solch eines Komplexes. Dem Einwand ausweichend könnte man "Warmphasenkomplex“ sagen. Das aber würde zwischen dem Rocourt-Solkomplex als Warmphasenkomplex und den schrittweise älteren Bodenkomplexen, dem Erft-Solkomplex und Rur-Solkomplex als Interglazialkomplexe (W. SCHIRMER 1999a), einen Gegensatz aufbauen, der dem Unterschied zu diesen nicht gerecht wird.

Man sollte daher definieren: Ein Interglazialkomplex ist eine Komplex aus Warmphasen und Breviglazialen, an dem wenigstens ein Interglazial per definitionem beteiligt ist, und der als Ganzes, Interstadiale und Brevigla- 
ziale mit einschließend, eine lange Zeit der Landschaftskonstanz verkörpert.

26) Schellmann (1990: 66) ordnet im Regensburger Raum vier schwache braune Böden dem Oberwürm zu.

\section{Literatur}

ANDERSEN, S. T. (1980): Early and Late Weichselian chronology and birch assemblages in Denmark. - Boreas, 9: 53-69; Oslo.

- Vries, H. de \& Zagwijn, W. H. (1960): Climatic change and radiocarbon dating in the Weichselian Glacial of Denmark and The Netherlands. - Geologie en Mijnbouw, 39: 38-42; s'Gravenhage.

App, V., Campen, J., Domsek, G. \& Hahn, J. (1987): Eine altsteinzeitliche Fundstelle auf dem Schwalbenberg bei Remagen, Kreis Ahrweiler (Vorbericht). - Trierer Z. f. Gesch. u. Kunst. d. Trierer Landes- und Nachbargebiete, Beih. 9: 85-102; Trier.

BAYER, J. (1927): Der Mensch im Eiszeitalter. - X + 452 S., 1 Farbtaf.; Leipzig, Wien (Deuticke).

Beaulieu, J.-L. De, Monjuvent, G. \& Nicoud, G. (1991): Chronology of the Würmian glaciation in the French Alps: a survey and new hypotheses. - Paläo klimaforschung, 1: 435-448; Stuttgart.

- \& Reille, MM. (1992): The last climatic cycle at La Grande Pile (Vosges, France). A new pollen profile. - Quaternary Sc. Rev., 11: 431-438; Oxford.

BeHre, K.-E. (1989): Biostratigraphy of the Last Glacial Period in Europe. - Quaternary Sc. Rev., 8: 25 - 44; Oxford.

- \& Lade, U. (1986): Eine Folge von Eem und 4 Weichsel-Interstadialen in Oerel/Niedersachsen und ihr Vegetationsablauf. - Eiszeitalter und Gegenwart, 36: 11-36, Taf. 1-2 als Beil.; Hannover.

- \& Plicht, J. VAN der (1992): Towards an absolute chronology for the last glacial period in Europe: radiocarbon dates from Oerel, northern Germany. Veget. Hist. Archaeobot., 1: 111-117; Berlin.

Bibus (1989), mit Beiträgen von W. RÄHLE und L. Zöller: 8. Tagung des Arbeitskreises „Paläoböden” der Deutschen Bodenkundlichen Gesellschaft vom 25. 5. - 27. 5. 1989 in Heilbronn. Programm und Exkursionsführer. - 31 S.; Tübingen (Geogr. Inst.).

Bock, W., Menke, B., Strehl, E. \& Ziemus, H. (1985): Neuere Funde des Weichselspätglazials in Schleswig-Holstein. - Eiszeitalter und Gegenwart, 35: 161-180; Hannover.

Bond, G., Broecker, W., Johnsen, S., McManus, J., LaBeyrie, L., Jouzel, J. \& Bonani, G. (1993): Correla tions between climate records from North Atlantic sediments and Greenland ice. - Nature, 365: 143147; London.

BüDEL, J. (1953): Die „periglazial”-morphologischen Wirkungen des Eiszeitklimas auf der ganzen Erde. Erdkunde, 7: 249-266; Bonn.

Chaline, J. \& Jerz, H. (1984): Arbeitsergebnisse der Subkommission für Europäische Quartärstratigraphie. Stratotypen des Würm-Glazials (Berichte der SEQS 6). - Eiszeitalter und Gegenwart, 35: 185-206; Hannover.

Dansgaard, W., Johnson, S. J., Clausen, H. B., Dahl-JenSen, D., Gundestrup, N. S., Hammer, C. U., Hvidberg,
C. S., Steffensen, J. P., Sveinbjörnsdottir, A. E., JouZEL, J. \& BOND, G. (1993): Evidence for general instability of past climate from a $250-\mathrm{kyr}$ ice-core record. - Nature, 364: 218-220; London.

- Johnsen, S. J., Clausen, H. B. \& Langway, C. C. jr. (1971): Climatic record revealed by the Camp Century ice core. - In: TuREkiAn, K. K. [ed.]: The Late Cenozoic glacial ages: 37-56; New Haven, London (Yale Univ. Press).

ELLWANGER, D. (1995): Rhein foreland glacier. - In: SCHIRMER, W. [ed.]: Quaternary field trips in Central Europe, 1: 506-511; München (Pfeil).

FeldmanN, L. (1996): Das Quartär im Harzvorland im Raum Schladen und Vienenburg nordöstlich von Goslar. - Exk.-Führer u. Veröff. GGW, 197: 182-192; Clausthal-Zellerfeld.

FINK, J. (1960): Leitlinien einer österreichischen Quartärstratigraphie. - Mitt. geol. Ges. Wien, 53: 249-266, 3 Taf.; Wien.

- (1964): Die Subkommission für Lößstratigraphie der Internationalen Quartärvereinigung. - Eiszeitalter und Gegenwart, 15: 229-235; Öhringen/Württ.

- (1969): Le loess en Autriche. - Bull. Ass. franç. étude Quaternaire, Supplement: La stratigraphie des loess d'Europe: 17-21; Paris.

- (1979): Stand und Aufgaben der österreichischen Quartärforschung. - Innsbrucker geogr. Studien, 5: 79-104; Innsbruck.

Frechen, M. \& Preusser, F. (1996): Kombinierte Lumineszenz-Datierungen am Beispiel des Lößprofils Mainz-Weisenau. - Frankfurter geowiss. Arb., D 20: 53-66; Frankfurt a. M.

Frenzel, B. (1991): Über einen frühen letzteiszeitlichen Vorstoß des Rheingletschers in das deutsche Alpenvorland. - Paläoklimaforschung, 1: 377-400; Stuttgart.

GöTzinger, G. (1935): Zur Gliederung des Lößes. Leimen- und Humuszonen im Viertel unter dem Manhartsberge. - Verh. geol. Bundesanstalt, 1935 (8/9): 126-132; Wien.

GRIP (Greenland Ice-core Project) Members (1993): Climate instability during the last interglacial period recorded in the GRIP ice core. - Nature, 364: 203207; London.

GRÜGER, E. (1979): Die Seeablagerungen vom Samerberg/Obb. und ihre Stellung im Jungpleistozän. Eiszeitalter und Gegenwart, 29: 23-34; Hannover.

- (1989): Palynostratigraphy of the last interglacial/glacial cycle in Germany. - Quaternary International, 3/4: 69-79; Oxford.

Gullentops, F. (1954): Contribution à la chronologie du Pléistocène et des formes du relief en Belgique. Mém. Inst. Geol. Univ. Louvain, 18: 123-252; Louvain.

Hammen, T. van der, Maarleveld, G. C., Vogel, J. C. \& ZaGWijn, W. H. (1967): Stratigraphy, climatic succession and radiocarbon dating of the Last Glacial in The Netherlands. - Geologie en Mijnbouw, 46 (3): 79-95; 's Gravenhage.

Hammer, C. U., Clausen, H. B., Dansgaard, W., Neftel, A., Kristins-Dottir, P. \& Johnson, E. (1985): Continuous impurity analysis along the Dye 3 Deep Core. - Geophysical Monograph Series, 33: 90-94; Washington D. C. 
Henze, N. (1998): Kennzeichnung des Oberwürmlösses in der Niederrheinischen Bucht. - Kölner Forum Geol. Paläont., 1: 212 S.; Köln [erschienen 1999].

Jouzel, J., Lorius, C., Petit, J. R., Genthon, C., Barkov, N. I., Kotlyakov, V. M. \& Petrov, V. M. (1987): Vostok ice core: a continuous isotope temperature record over the last climatic cycle (160.000 years). Nature, 329: 403-407; London.

Keller, O. \& Krayss, E. (1998): Datenlage und Modell einer Rhein-Linth-Vorlandvergletscherung zwischen Eem-Interglazial und Hochwürm. - In: IKINGER, A. [Hrsg.]: Festschrift Wolfgang Schirmer. Geschichte aus der Erde. - GeoArchaeoRhein, 2: 121-137; Münster.

Krayss, E. (1988): Zur riß-würmzeitlichen Quartärgeologie im westlichen Rheingletschergebiet. - Z. Geomorph. N. F., Suppl.-Bd. 70: 1-12; Berlin.

LundQvist, J. (1974): Outlines of the Weichsel Glacial in Sweden. - Geologiska Föreningens i Stockholm Verhandlingar, 96: 327-339; Stockholm.

MaKOwsKA, A. (1975): Die Früh-Würm-Kaltzeit in Nordpolen. - Wiss. Z. Ernst-Moritz-Arndt-Universität Greifswald, 24, Math.-naturwiss. Reihe, 3/4: 135141; Greifswald.

Mangerud, J. (1991): The Scandinvian ice sheet through the last interglacial/glacial cycle. - Paläoklimaforschung, 1: 307-330; Stuttgart.

Marks, L., Piotrowski, J. A., Stephan, H.-J., Fedorowicz, S. \& Butrym, J. (1995): Thermoluminescence indications of the Middle Weichselian (Vistulian) Glaciation in northwest Germany. - Meyniana, 47: 6982; Kiel

McManus, J. F., Bond, G. C., Broecker, W. S., Johnsen, S., Labeyrie, L. \& Higgins, S. (1994): High-resolution climate records from the North Atlantic during the last interglacial. - Nature, 371: 326-329; London.

Mejus, E., Mücher, H., Ouwerkerk, G., Romein, A. \& StolTENBERG, H. (1983): Evidence of the presence of the Eltville Tuff layer in Dutch and Belgian Limbourg and the consequences for the loess stratigraphy. Eiszeitalter und Gegenwart, 33: 59-78, Fig. 7 als Falttaf.; Hannover.

MEnke, B. (1976): Neue Ergebnisse zur Stratigraphie und Landschaftsentwicklung im Jungpleistozän Westholsteins. - Eiszeitalter und Gegenwart, 27: 5368, Öhringen.

- (1980): Keller (nordwestlich von Schenefeld), EemInterglazial und Weichsel-Frühglazial. - In: STREMME, H. E. \& MENkE, B. [Hrsg.]: Quartär-Exkursionen in Schleswig-Holstein: 35-49; Kiel (Geol. Landesamt Schleswig-Holstein).

Müller-Beck, H. (1959): Bemerkungen zur Stratigraphie des mitteleuropäischen Jungpleistozäns. - Eiszeitalter und Gegenwart, 10: 144-160; Öhringen/Württ.

Paterne, M., Guichard, F., Labeyrie, J., Gillot, P. Y. \& Duplessy, J. C. (1986): Tyrrhenian Sea tephrochronology of the oxygen isotope record for the past 60.000 years. - Marine Geology, 72: 259-285; Amsterdam.

Petersen, K. S. (1984): Stratigraphical position of Weichselian tills in Denmark. - Striae, 20: 75-78; Uppsala.

Pistas, N. G., Martinson, D. G., Moore, T. C. Jr., Shackleton, N. J., Prell, W., Hays, J. \& Boden, G. (1984): High resolution stratigraphic correlation of benthic oxygen isotopic records spanning the last 300,000 years. - Marine Geology, 56: 119-136; Amsterdam.

Schellmann, G. (1990): Fluviale Geomorphodynamik im jüngeren Quartär des unteren Isar- und angrenzenden Donautales. - Düsseldorfer geogr. Schr., 29: 131 S.; Düsseldorf.

Schiermeyer, J. (2000): Würmzeitliche Lößmollusken aus der Eifel. - Inaug.-Diss. Univ. Düsseldorf: 125 S.; Düsseldorf. Veröffentlicht unter: http://www.ulb.uni-duesseldorf.de/diss/mathnat/2000/schiermeyer.html

Schirmer, U. (1995): Early Late Glacial pollen record of Miesenheim. - In: SCHIRMER, W. [ed.]: Quaternary field trips in Central Europe, 1: 533-535; München (Pfeil). (1996): Pollen stratigraphy below the Pellenz tephra. - In: Schirmer, W., Ikinger, A., Schiermeyer, J., Schirmer, U. \& WALDMANN, G. : Guide to the archives of the Laacher See eruptions: 15-17; Düsseldorf (Dept. Geol. HHUniversity).

- (1999): Pollenstratigraphische Gliederung des Spätglazials im Rheinland. - Eiszeitalter und Gegenwart, 49: 132-143; Hannover.

SCHIRMER, W. (1990): Schwalbenberg südlich Remagen. - In: Schirmer, W. [Hrsg.]: Rheingeschichte zwischen Mosel und Maas. - deuqua-Führer, 1: 105-108; Hannover (DEUQUA).

- (1991): Würmzeitliche Paläoböden am Mittelrhein. 10. Tagung des Arbeitskreises Paläoböden der Deutschen Bodenkundlichen Gesellschaft vom 30. 5. - 1. 6. 1991 in Bonn, Programm und Exkursionsführer: 70-83; Münster.

- (1992): Doppelbodenkomplexe in Erkelenz und Rheindahlen. - In: Arbeitskreis Paläopedologie [Hrsg.]: Bodenstratigraphie im Gebiet von Maas und Niederrhein: 86-94; Kiel (Dt. Bodenkdl. Ges.).

- (1995a): Mittelrhein Basin and lower Mittelrhein. In: SCHIRmer, W. [ed.]: Quaternary field trips in Central Europe, 1: 524-537; München (Pfeil).

- (1995b): Niederrhein Bay. - In: Schirmer, W. [ed.]: Quaternary field trips in Central Europe, 1: 537-545; München (Pfeil).

- (1995c): The Oberrheingraben and its borders. - In: SChirmer, W. [ed.]: Quaternary field trips in Central Europe, 1: 511-520; München (Pfeil).

- (1995d): Valley bottoms in the late Quaternary. - Z. Geomorph. N. F., Suppl.-Bd. 100: 27-51; Berlin.

(1996): Spätglaziale Böden unter Laacher See-Tephra. - In: Landesamt für Natur und Umwelt des Landes Schleswig-Holstein [Hrsg.]: Böden als Zeugen der Landschaftsentwicklung: 49-58; Kiel (L.-A. Natur u. Umwelt SH).

(1998): Eine Klimakurve aus dem rheinischen Löß der letzten Kaltzeit. - In: Feldmann, L., Benda, L. \& LOOK, E.-R. [Hrsg.]: DEUQUA Jubiläums-Hauptversammlung in Hannover, 13. bis 20. September 1998. Kurzfassungen der Vorträge und Poster: 51; [Hannover].

- (1999a): Kaltzeiten und Warmzeiten im Löß. - In: Becker-Haumann, R. \& Frechen, M. [Hrsg.]: Terrestrische Quartärgeologie: 81-100; Köln (Logabook).

- (1999b): Garzweiler 4 - eine Stecknadel im Heuhaufen der letzten Warmzeit und Eiszeit. - Archäologie im Rheinland, 1998: 149-152; Köln. 
- (1999c): Dune phases and soils in the European sand belt. - GeoArchaeoRhein, 3: 11-42; Münster.

(1999d): Climate and stratigraphy of the Last Glacial in the Rheinland. - In: Neanderthal Museum: Central and Eastern Europe from 50.000-30.000 B.P. International Whorkshop in the Neanderthal Museum, March 18-21, 1999. Abstracts: 16-17; Neanderthal-Museum.

- (1999e): O-stages 2-5 in the Rhein loess, ice cores and deap-sea cores. - Int. Union Quaternary Res., 15. Int. Congr. 3-11 Aug. 1999, Durban, South Africa. The environmental background to hominid evolution in Africa. Book of Abstracts: 158-159; Durban. - Ebenso: Quaternary Int., 63/64: 129; 2000. [zugehörige Abb. wurden in beiden Drucken nicht dargestellt]

- (2000a): Rhein loess, ice cores and deep-sea cores during MIS 2-5. - Z. dt. geol. Ges., 151 (3): 309-332; Stuttgart.

- (2000b): Die Eben-Zone im Oberwürmlöss zwischen Maas und Rhein. - In: SCHIRMER, W. [Hrsg.]: Landschaftsgeschichte im europäischen Rheinland. [in Druckvorbereitung]

- \& Feldmann, L. (1992): Das Lößprofil von Rheindahlen/Niederrhein. - In: Arbeitskreis Paläopedologie [Hrsg.]: Bodenstratigraphie im Gebiet von Maas und Niederrhein: 76-85; Kiel (Dt. Bodenkdl. Ges.).

- \& STReIT, R. (1967): Die Deckschichten der niederrheinischen Hauptterrasse bei Erkelenz. - Sonderveröff. Geol. Inst. Univ. Köln, 13: 81-94; Köln.

Schlüchter, C. (1991): Fazies und Chronologie des letzteiszeitlichen Eisaufbaus im Alpenvorland der Schweiz. - Paläoklimaforschung, 1: 401-407; Stuttgart.

Schönhals, E., Rohdenburg, H. \& Semmel, A. (1964): Ergebnisse neuerer Untersuchungen zur WürmlößGliederung in Hessen. - Eiszeitalter und Gegenwart, 15: 199-206; Öhringen.

Seidenkrantz, M.-S. \& KNudsen, K. L. (1997): Eemian climatic and hydrographical instability on a marine shelf in Northern Denmark. - Quaternary Research, 47: 218-234; Washington.

Semmel, A. (1995): Die quartären Deckschichten im Dyckerhoff-Steinbruch am Kinzenberg westlich Wiesbaden-Erbenheim. - Geol. Jb. Hessen, 123: 133-137; Wiesbaden.

- (1996): Paläoböden im Würmlöß, insbesondere im Altwürmlöß des Steinbruchs Mainz-Weisenau - Problemstellung und Übersicht über die Forschungsergebnisse. - Frankfurter geowiss. Arb., D 20: 11-20; Frankfurt a. M.

- (1998a): Zur paläopedologischen Gliederung des älteren Würmlösses in Mitteleuropa. - Mitt. dt. bodenkdl. Ges., 88: 449-452; Oldenburg.

(1998b): Lokalitäten von Stratotypen jungpleistozäner äolischer und solifluidaler Sedimente im RheinMain-Gebiet. - In: IKINGER, A. [Hrsg.]: Festschrift Wolfgang Schirmer. Geschichte aus der Erde. GeoArchaeoRhein, 2: 35-45; Münster (Lit Verlag).

(1999): Die paläopedologische Gliederung des älteren Würmlösses in Mitteleuropa - erörtert an Beispielen aus dem Rhein-Main-Gebiet. - Z. geol. Wiss., 27 (1/2): 121-133; Berlin.
Staalduinen, C. J. van, Adrichem Boogaert, H. A. van, Bless, M. J. M., Doppert, J. W. C., Harsveldt, H. M., Montfrans, H. M. van, Oele, E., Wermuth, R. A. \& ZaGwIJN, W. H. (1979): The Geology of The Netherlands. - Meded. Rijks Geol. Dienst, 31 (2): 9-49; Haarlem.

Thouveny, N., Beaulieu, J.-L. De, Bonifay, E., Creer, K. M., Guiot, J., Icole, M., Johnsen, S., Jouzel, J., Reille, M., Williams, T. \& Williamson, D. (1994): Climate variations in Europe over the past $140 \mathrm{kyr}$ deduced from rock magnetism. - Nature, 371: 503-506; London.

VAndenberghe, J. (1985): Paleoenvironment and stratigraphy during the Last Glacial in the Belgian-Dutch border region. - Quaternary Research, 24: 23-38; New York.

WeLteN, M. (1981): Verdrängung und Vernichtung der anspruchsvollen Gehölze am Beginn der letzten Eiszeit und die Korrelation der Frühwürm-Interstadiale in Mittel- und Nordeuropa. - Eiszeitalter und Gegenwart, 31: 187-202; Hannover.

- (1982): Stand der palynologischen Quartärforschung am schweizerischen Nordalpenrand (Überblick, Methodisches, Probleme). - Geogr. Helv., 37 (2): 75-83; Bern.

Woillard, G. M. (1978): Grande Pile peat bog: A continuous pollen record for the last 140,000 years. Quaternary Research, 9: 1-21; New York.

- (1979): The last interglacial-glacial cycle at Grand Pile in Northeastern France. - Bull. Soc. belge de Géologie, 88 (1): 51-69; Bruxelles.

- \& Mоок, W. G. (1982): Carbon-14 dates at Grande Pile: Correlation of land and sea chronologies. Science, 215: 159-161; Washington D. C.

Woldstedt, P. (1954): Saaleeiszeit, Warthestadium und Weichseleiszeit in Norddeutschland. - Eiszeitalter und Gegenwart, 4/5: 34-48; Öhringen/Württ.

- (1956): Über die Gliederung der Würm-Eiszeit und die Stellung der Lösse in ihr. - Eiszeitalter und Gegenwart, 7: 78-86, 1 Abb.; Öhringen.

- (1958a): Eine neue Kurve der Würm-Eiszeit. - Eiszeitalter und Gegenwart, 9: 151-154; Öhringen.

- (1958b): Das Eiszeitalter. Grundlinien einer Geologie des Quartärs, 2, 2. Aufl.: 433 S., Taf. 1; Stuttgart (Enke).

- (1960): Die letzte Eiszeit in Nordamerika und Europa. - Eiszeitalter und Gegenwart, 11: 148-165; Öhringen.

- (1962): Über die Gliederung des Quartärs und Pleistozäns. - Eiszeitalter und Gegenwart, 13: 115-124; Öhringen.

Wunstorf, W. (1913): Über Löß und Schotterlehm im Niederrheinischen Tiefland. - Verh. naturhist. Ver. preuss. Rheinlande u. Westfalen, 69, 1912: 293-340; Bonn.

ZaGWiJn, W. \& PAepe, R. (1968): Die Stratigraphie der weichselzeitlichen Ablagerungen der Niederlande und Belgiens. - Eiszeitalter und Gegenwart, 19: 129-146; Öhringen.

Manuskript eingeg. am 10. März 1999,

begutachtet am 09. März 2000,

letzte Nachträge: 31. August 2000. 